\title{
Palladium and radical routes to phenanthridines
}

\author{
W. Russell Bowman, Jessica E. Lyon, and Gareth J. Pritchard* \\ Department of Chemistry, University of Loughborough, \\ Loughborough, Leicestershire, LE11 3TU, UK \\ E-mail: G.J.Pritchard@lboro.ac.uk
}

\section{We wish to dedicate this paper to mark Professor Keith Smith's retirement}

\begin{abstract}
Two routes to phenanthridines are reported; a palladium-mediated route using imidoyl-selanides as precursors and a modified radical route using aryl imines as starting materials.
\end{abstract}

Keywords: Phenanthridines, palladium, imines, radicals, imidoyl-selanides

\section{Introduction}

Phenanthridines are an important class of heterocyclic compounds first discovered in 1891 by Pictet and Ankersmit. They show a range of biological activities, ${ }^{1}$ and are currently attracting significant interest from synthetic chemists. The majority of established synthetic routes utilise anionic ring closure reactions, ${ }^{2}$ Bischler-Napieralski reactions, ${ }^{3}$ reduction of phenanthridones, ${ }^{4}$ palladium chemistry, ${ }^{5}$ or free radical methodology. ${ }^{6,7}$ A number of new syntheses of the phenanthridines have been reported recently, underlining their importance. ${ }^{8}$

Recently we have published a palladium-mediated route to phenanthridine, ${ }^{9}$ as part of our interest in the development of new methods to prepare heterocyclic systems which include stoichiometric organometallics, ${ }^{10}$ biomimetic methods, ${ }^{11}$ condensation of reactive electrophilic systems, ${ }^{12}$ and radical chemistry. ${ }^{13}$ We now wish to disclose the full details of our palladium work together with an improved phenanthridine synthesis by a radical method from imines.

\section{Results and Discussion}

Our first proposed route to phenanthridines 1 involves the key $\mathrm{Pd}$-mediated $\mathrm{C}-\mathrm{C}$ bond formation of the central pyridine ring from the imidoyl-selanides $\mathbf{2}$. The imidoyl-selanides $\mathbf{2}$ can in turn be derived from the corresponding amides $\mathbf{3}$ which are readily prepared from commercially 
available 2-aminobiphenyl 4 by well-established amide bond formation chemistry, such as simple reaction with aromatic acid chlorides (Scheme 1). We chose imidoyl selanides because the weak imidoyl-Se bond was predicted to facilitate $\operatorname{Pd}(0)$ insertion and they had proved good precursors for radical reactions. ${ }^{14}$ Also it has been shown that transition metals can insert into the carbon-selenium bond. ${ }^{15}$<smiles>[R]c1ccc(-c2nc3ccccc3c3ccccc23)cc1</smiles>

Scheme 1. Proposed synthesis of phenanthridines 1.

The required amides 3 were prepared, in reasonable yields, by standard methods from 2aminobiphenyl 4 and aryl acid chlorides (Scheme 2, Table 1). A representative range of 4substituted aryl acid chlorides was chosen to prepare the amides $\mathbf{3}$, in order to observe the electronic effect on the insertion of palladium into the Se-C bond of the imidoyl-selanides 2 (Table 1). The amides 3 were converted into the appropriate $\alpha$-chloroimines by treatment with phosgene and catalytic DMF. ${ }^{16}$ We found other methods of preparing the $\alpha$-chloroimines were unsatisfactory for further reactions. The potassium salt of phenylselanide was prepared in situ by reduction of diphenyl diselanide with $\mathrm{K}$-Selectride ${ }^{\circledR}$ and added directly to the un-purified $\alpha$ chloroimines to give the imidoyl-selanides 2 (Scheme 2, Table 1). ${ }^{14}$ Some hydrolysis of the imidoyl-selanides $\mathbf{2}$ was observed upon both silica and alumina chromatography, therefore the imidoyl-selanides $\mathbf{2}$ were used either after purification by crystallization, or rapid chromatography, and in some cases as the crude mixture.<smiles>[R]c1ccc(C(=O)Nc2ccccc2-c2ccccc2)cc1</smiles>

3a-3h

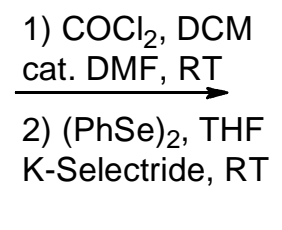

2a-2h

Scheme 2. Synthetic route to imidoyl-selanides $\mathbf{2 a - 2 h}$. 
Table 1. Yields of amides 3a-3h and imidoyl-selanides $\mathbf{2 a - 2 h}$

\begin{tabular}{cccc}
\hline Derivative & R Group & Amide 3 Yield (\%) & Selanide 2 Yield (\%) \\
\hline a & $\mathrm{H}$ & 52 & 54 \\
$\mathrm{~b}$ & $\mathrm{Me}$ & 64 & 44 \\
$\mathrm{c}$ & $t-\mathrm{Bu}$ & 59 & 66 \\
$\mathrm{~d}$ & $\mathrm{OMe}$ & 70 & 51 \\
$\mathrm{e}$ & $\mathrm{Cl}$ & 64 & 60 \\
$\mathrm{f}$ & $\mathrm{CF}_{3}$ & 77 & 60 \\
$\mathrm{~g}$ & $\mathrm{NMe}_{2}$ & 61 & 62 \\
$\mathrm{~h}$ & $\mathrm{NO}_{2}$ & 47 & Decomposed \\
\hline
\end{tabular}

With an array of imidoyl-selanides 2 in hand we attempted the proposed Pd-mediated cyclisation to phenanthridines 1. A large range of conditions have been reported for palladium catalyzed reactions, ${ }^{17}$ so in order to limit the number of variables in the preliminary reactions we initially used the conventional $\mathrm{Pd}\left(\mathrm{PPh}_{3}\right)_{4}$ as the palladium source without additional ligands. It is foreseeable that other palladium sources, or the inclusion of ligands, will also be applicable and we are keen to explore their potential in due course.

The parent imidoyl-selanide $(\mathrm{R}=\mathrm{H}) \mathbf{2 a}$ was first treated with $10 \% \mathrm{Pd}\left(\mathrm{PPh}_{3}\right)_{4}$ and excess triethylamine under a range of trial reaction conditions. In DCM, THF, or MeCN, no reactions took place at either room temperature or at reflux. Complex mixtures were observed when the reactions were carried out at $80{ }^{\circ} \mathrm{C}$ in DMF or chlorobenzene, with no discernible sign of the desired product. However, when the reaction was carried out at $80{ }^{\circ} \mathrm{C}$ in the non-polar solvent, toluene, a trace of the desired phenanthridine 1a was detected in the crude mixture. The ${ }^{1} \mathrm{H}$ NMR spectrum of the crude mixture clearly showed the characteristic peaks of the phenanthridine ring. Encouraged by this result the reaction was repeated at reflux in toluene for 48 hours which gave a $10 \%$ isolated yield. A further improvement in yield was seen when the amount of palladium catalyst was increased to 0.4 equiv. With these positive conditions in hand, the range of imidoylselanides 2a-2g previously prepared were treated with $\mathrm{Pd}\left(\mathrm{PPh}_{3}\right)_{4}(0.4$ equiv.) and excess triethylamine in toluene at reflux for 48 hours, to successfully produce the respective phenanthridines 1a-1g (Scheme 3, Table 2).

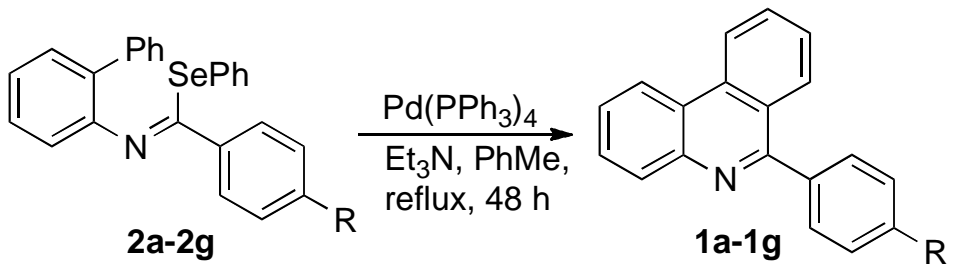

Scheme 3. Pd-mediated cyclisation to phenanthridines 1a-1g. 
Table 2. Yields of phenanthridines 1a-1g

\begin{tabular}{ccc}
\hline Phenanthridine & R Group & Yield (\%) \\
\hline $1 \mathrm{a}$ & $\mathrm{H}$ & 28 \\
$1 \mathrm{~b}$ & $\mathrm{Me}$ & 34 \\
$1 \mathrm{c}$ & $t$-Bu & 47 \\
$1 \mathrm{~d}$ & $\mathrm{OMe}$ & 39 \\
$1 \mathrm{e}$ & $\mathrm{Cl}$ & 46 \\
$1 \mathrm{f}$ & $\mathrm{CF}_{3}$ & 48 \\
$1 \mathrm{~g}$ & $\mathrm{NMe}_{2}$ & 22 \\
\hline
\end{tabular}

The phenanthridines 1a-1g were isolated in synthetically useful yields that are currently unoptimised. The electronic effect of the substitution on the aromatic ring (R) did not appear to affect the reaction. In the absence of the base there was no reaction or significantly reduced yield. Attempts to cyclise the $\alpha$-chloroimines, precursors of the imidoyl-selanides, under all conditions failed to give any signs of the phenanthridines in our hands to date. We are pleased with these initial results and a wider range of targets is currently under investigation using imidoyl-selanides and Pd-mediated conditions. We are also looking at optimising the reaction conditions by using a different base, palladium source and ligands that are known to enhance Pdcatalysis.

Mechanistically, we propose insertion of a $\operatorname{Pd}(0)$ species into the carbon selenium bond followed by carbo-palladation onto the phenyl ring. This intermediate then undergoes rapid rearomatization with the loss of $\mathrm{HPdSePh}$ to give the phenanthridine system (Scheme 4).<smiles>[R]c1ccc(C(=Nc2ccccc2P)c2ccccc2)cc1</smiles><smiles>[R]c1ccc(C2=Nc3ccccc3C3=CC=CC(POc4ccccc4)C32)cc1</smiles>

Scheme 4. Proposed mechanism of Pd-mediated cyclisation of phenanthridine 1.

Incidentally, while we were looking up spectral data of the phenanthridines prepared using the previously described novel palladium chemistry, we became aware of some leading work by 
Leardini et al. in the 1980s. ${ }^{7}$ These workers had shown under radical conditions imines were suitable precursors to a range of phenanthridines (Scheme 5). Using di-iso-propyl peroxy carbonate (DPDC) they showed that a range of imines (Scheme 5) could be cyclised to give phenanthridines in good yields, in hot benzene. The reaction proceeds by initial imidoyl-H atom abstraction by the electrophilic $i$-PrO radical, and subsequently the intermediate undergoes intramolecular cyclization and oxidative aromatization to form the phenanthridine ring.

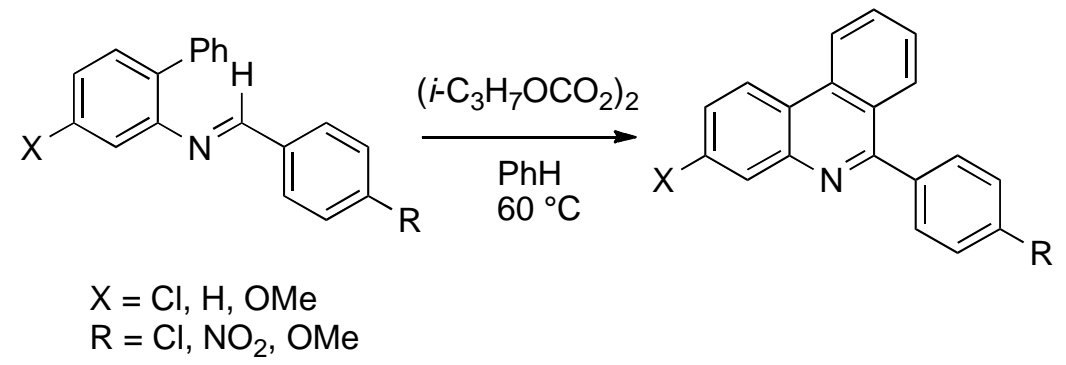

Scheme 5. Radical reaction reported by Leardini et al.

With our experience in radical chemistry, we decided to enhance this methodology as part of our interests in the field of phenanthridines. We considered that the use of di-iso-propyl peroxycarbonate (DPDC) is somewhat of a drawback due to its explosive nature, thus requiring additional safety precautions and limiting its potential application. ${ }^{18}$ In place of di-iso-propyl peroxycarbonate (DPDC), we planned to use di-(tert-butyl)peroxide, which is reported not to be explosive under normal conditions, as a safer alternative. ${ }^{18}$ Homolysis of the di-(tertbutyl)peroxide, which can be facilitated by heat, yields the strongly electrophilic radical $t$-BuO', which should be an ideal substitute for $i$-PrO and a similar reaction followed.

The desired aryl imines were readily prepared by standard methods in decent yields. 2Aminobiphenyl 4 and an aryl aldehyde were stirred together in dichloromethane in the presence of molecular sieves at room temperature to give the required imines $\mathbf{5 a - 5 h}$ (Scheme 6, Table 3).

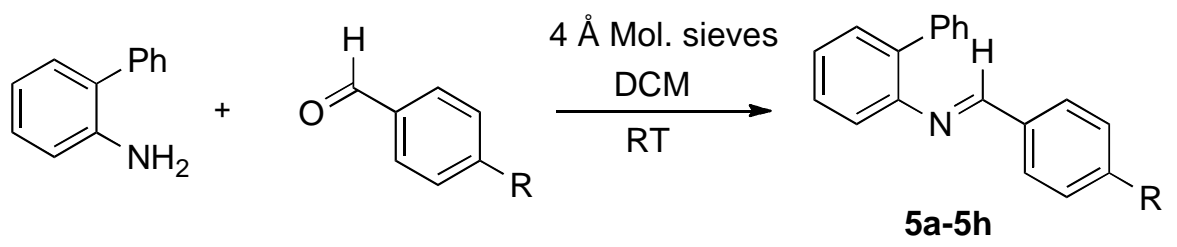

Scheme 6. Preparation of imines 5a-5h.

With the imines in hand we could attempt the improved radical conditions for the reaction. Homolysis of the di-(tert-butyl)peroxide occurs at $120{ }^{\circ} \mathrm{C}$, therefore a high boiling point solvent was required. Our initial attempts to cyclise the imine 5a was carried out in chlorobenzene using two equivalents of the peroxide at $125{ }^{\circ} \mathrm{C}$. The ${ }^{1} \mathrm{H}$ NMR spectrum showed no trace of the desired 
product. The boiling point of di-(tert-butyl)peroxide is $109{ }^{\circ} \mathrm{C}$ and it was therefore thought that homolysis was not occurring or was undergoing evaporation prior to homolysis. This problem was solved by repeating the reaction in a sealed vessel and heating the reaction mixture to 140$150{ }^{\circ} \mathrm{C}$ (oil bath temperature) which led to the desired product in $48 \%$ yield. The purified phenanthridine showed an identical ${ }^{1} \mathrm{H}$ NMR spectrum to the sample previously prepared by $\mathrm{Pd}$ catalysis. The un-optimised yield was somewhat lower that reported by Leardini using DPDC, but this does provide a safer alternative especially when scaling up.

Table 3. Yields of imines $\mathbf{5 a - 5 h}$

\begin{tabular}{ccc}
\hline Imine & $\mathrm{R} \mathrm{Group}$ & Yield (\%) \\
\hline $\mathbf{5 a}$ & $\mathrm{H}$ & 60 \\
$\mathbf{5 b}$ & $\mathrm{Me}$ & 66 \\
$\mathbf{5 c}$ & $t$-Bu & 68 \\
$\mathbf{5 d}$ & $\mathrm{OMe}$ & 78 \\
$\mathbf{5 e}$ & $\mathrm{Cl}$ & 79 \\
$\mathbf{5 f}$ & $\mathrm{CF}_{3}$ & 58 \\
$\mathbf{5 g}$ & $\mathrm{NMe}_{2}$ & 66 \\
$\mathbf{5 h}$ & $\mathrm{NO}_{2}$ & 86 \\
\hline
\end{tabular}

With this gratifying result in-hand we decided to look at a range of substituents on the aldehyde component of the reaction. The choice of a $p$-substituted benzaldehyde reflects the possible influence of electronic effects and also parallels our palladium chemistry. In addition it expands on the limited range of examples Leardini had reported. All the imines were prepared in reasonably yields (Table 4) and used shortly after preparation to avoid potential hydrolysis or oxidation problems. The imines $\mathbf{5 b} \mathbf{b}-\mathbf{5 h}$ were submitted to the radical cyclisation conditions, di(tert-butyl)peroxide (2 equiv.) in chlorobenzene at $140-150{ }^{\circ} \mathrm{C}$ for $48 \mathrm{~h}$, to yield the corresponding phenanthridines $\mathbf{1 b}-\mathbf{1 h}$ in moderate yields (Scheme 7, Table 4). There was no significant difference in the results with respect to the effect of the functional group on the cyclisation.

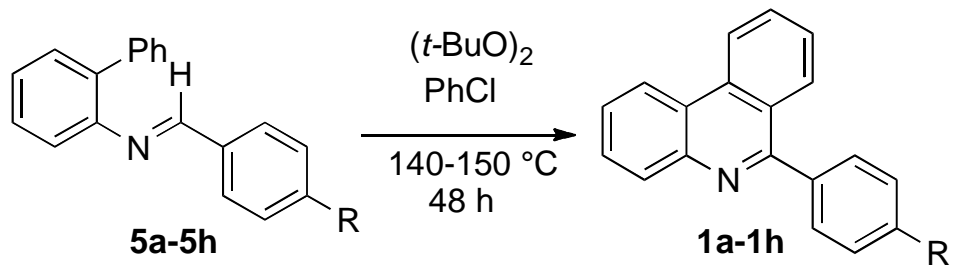

Scheme 7. Synthesis of phenanthridines $\mathbf{1 a - 1 h}$ by radical cyclisation. 
Table 4. Yields of phenanthridines 1a-1h

\begin{tabular}{ccc}
\hline Phenanthridine & $\mathrm{R} \mathrm{Group}$ & Yield (\%) \\
\hline 1a & $\mathrm{H}$ & 40 \\
$\mathbf{1 b}$ & $\mathrm{Me}$ & 44 \\
1c & $t-\mathrm{Bu}$ & 39 \\
1d & $\mathrm{OMe}$ & 50 \\
1e & $\mathrm{Cl}$ & 48 \\
1f & $\mathrm{CF}_{3}$ & 51 \\
1g & $\mathrm{NMe}_{2}$ & 42 \\
1h & $\mathrm{NO}_{2}$ & 46 \\
\hline
\end{tabular}

The mechanism for the formation of the phenanthridines is that of homolytic aromatic substitution. ${ }^{19}$ The $t$-BuO radical abstracts the imine- $H$ forming the imidoyl radical, which then adds to the phenyl ring. The homolytic aromatic substitution is then terminated by $\mathrm{H}$-atom abstraction by another radical such as a $t$-BuO radical.<smiles>[R]c1ccc(C(=Nc2ccccc2-c2ccccc2)c2ccccc2-c2ccccc2)cc1</smiles><smiles>[R]c1ccc(-c2nc3ccccc3c3ccccc23)cc1</smiles>

Scheme 8. Proposed homolytic aromatic substitution mechanism of phenanthridine $\mathbf{1}$ formation. Conclusions

We are pleased with the initial palladium results and the modified radical reaction conditions for the preparation of phenanthridines, but there is plenty of scope for optimising both the radical and palladium reaction conditions, such as using alternative palladium sources, the addition of ligands, and different base/solvent combinations. Currently, we are investigating further chemistry of the imidoyl-selanides/palladium methodology to synthesise a wide range of heterocyclic targets other than phenanthridines. Moreover, our research into the development of 
new palladium reactions and radical reactions to the same target could lead to a valuable direct comparison of radical and palladium reactions in the generation of important heterocyclic compounds.

\section{Experimental Section}

General. Infrared spectra were obtained using a Perkin-Elmer Paragon 1000 FT-IR spectrophotometer in the range of $4000-600 \mathrm{~cm}^{-1}$ (with internal calibration). Samples were dissolved in the appropriate solvent and applied to sodium chloride plates as thin films. In the case of liquid samples, they were applied neat to the plates and run as thin films. Only the major absorbances have been quoted.

${ }^{1} \mathrm{H}$ NMR spectra were measured at $400 \mathrm{MHz}$ and ${ }^{13} \mathrm{C}$ NMR spectra were measured at $100 \mathrm{MHz}$ (unless stated otherwise), using a Bruker AC $400 \mathrm{MHz}$ spectrometer. The solvent used for spectroscopy was $\mathrm{CDCl}_{3}$ (unless stated otherwise) using TMS as the internal reference. Chemical shifts are given in part per million (ppm) and coupling constants ( $J$ values) are given in hertz $(\mathrm{Hz})$. Assignment of individual proton signals was assisted by analysis of ${ }^{1} \mathrm{H}$ COSY spectra.

Melting points were obtained using an electrical 9100 Thermal melting point instrument and are uncorrected.

All solvents and reagents were purified by standard technique or used as supplied from chemical sources as appropriate. Light petrol refers to the fraction which boils at $40-60{ }^{\circ} \mathrm{C}$ Reagent chemicals were purchased from Aldrich Chemical Company Ltd, Lancaster Chemical Synthesis Ltd and Acros (Fischer) Chemicals Ltd. Solvents when necessary were dried and stored over $4 \AA$ molecular sieves prior to use.

\section{General experimental procedure for the preparation of the benzamides 3}

To a solution of 2-aminobiphenyl 4 (1.0 equiv.) and $\mathrm{Et}_{3} \mathrm{~N}$ (2.0 equiv.) in $\mathrm{CH}_{2} \mathrm{Cl}_{2}(0.3 \mathrm{ml} / \mathrm{mmol})$ at $0{ }^{\circ} \mathrm{C}$ was added acid chloride (1.0 equiv.). The reaction was warmed to $\mathrm{rt}$ and stirred for $5 \mathrm{~h}$. The reaction mixture was then washed with water, sat. $\mathrm{NaHCO}_{3}$ solution, and brine. The organics were dried over $\mathrm{MgSO}_{4}$, filtered and concentrated under reduced pressure. The residue was purified by trituration with DCM and hexanes. The resulting solid was filtered off and dried under vacuum to yield pure benzamide 3 .

$N$-Biphenyl-2-yl benzamide (3a). Benzoyl chloride $(0.34 \mathrm{ml}, 2.95 \mathrm{mmol})$ yielded $N$-biphenyl-2yl benzamide 3a $(419 \mathrm{mg}, 52 \%)$ as off-white crystals mp $87-88{ }^{\circ} \mathrm{C} . \delta_{\mathrm{H}}\left(400 \mathrm{MHz}, \mathrm{CDCl}_{3}\right) 7.43$ $(13 \mathrm{H}, \mathrm{m}, \mathrm{Ar}-H), 8.00(1 \mathrm{H}, \mathrm{s}, \mathrm{NH}), 8.54(1 \mathrm{H}, \mathrm{d}, J$ 8.4, Ar- $H) ; \delta_{\mathrm{C}}\left(100 \mathrm{MHz}, \mathrm{CDCl}_{3}\right) 121.1(\mathrm{Ar}-$ $\mathrm{CH}), 124.4(\mathrm{Ar}-\mathrm{CH}), 126.8(2 \times \mathrm{Ar}-\mathrm{CH}), 128.2(\mathrm{Ar}-\mathrm{CH}), 128.6(\mathrm{Ar}-\mathrm{CH}), 128.7$ ( 2 x Ar- $\mathrm{CH})$, 129.2 ( 2 x Ar- $\mathrm{CH}), 129.4$ (2 x Ar- $\mathrm{CH}), 130.0$ (Ar- $\mathrm{CH}), 131.7$ (Ar- $\mathrm{CH}), 132.3(\mathrm{Ar}-\mathrm{C}), 134.8$ (Ar$C), 134.9(\mathrm{Ar}-C), 138.0(\mathrm{Ar}-C), 165.0(C=\mathrm{O})$; HRMS $\left(\mathrm{FAB}^{+}\right)$Found: $\left[\mathrm{M}+\mathrm{H}^{+}\right] 274.1234$. $\mathrm{C}_{19} \mathrm{H}_{16} \mathrm{NO}$ requires 274.1232; $\mathrm{m} / z\left(\mathrm{FAB}^{+}\right) 274$ ([M+H $\left.\left.{ }^{+}\right], 73 \%\right), 273$ (46), 105 (100), 77 (25). 
$N$-Biphenyl-2-yl-4-methyl benzamide (3b). Toluoyl chloride $(0.39 \mathrm{ml}, 2.95 \mathrm{mmol})$ yielded $N$ biphenyl-2-yl benzamide $3 \mathbf{b}(540 \mathrm{mg}, 64 \%)$ as off-white crystals mp $108-109{ }^{\circ} \mathrm{C} . v_{\max }(\mathrm{KBr}) / \mathrm{cm}^{-}$ ${ }^{1} 3190,1620,1590 ; \delta_{\mathrm{H}}\left(400 \mathrm{MHz}, \mathrm{CDCl}_{3}\right) 2.37\left(3 \mathrm{H}, \mathrm{s}, \mathrm{CH}_{3}\right), 7.23(4 \mathrm{H}, \mathrm{m}, \mathrm{Ar}-H), 7.47(8 \mathrm{H}, \mathrm{m}$, Ar- $H), 7.98(1 \mathrm{H}, \mathrm{s}, \mathrm{NH}), 8.54(1 \mathrm{H}, \mathrm{dd}, J 0.8,8, \mathrm{Ar}-H) ; \delta_{\mathrm{C}}\left(100 \mathrm{MHz}, \mathrm{CDCl}_{3}\right) 21.4\left(\mathrm{CH}_{3}\right), 121.0$ $(\mathrm{Ar}-\mathrm{CH}), 124.2(\mathrm{Ar}-\mathrm{CH}), 126.8(2 \times \mathrm{Ar}-\mathrm{CH}), 128.1(\mathrm{Ar}-\mathrm{CH}), 128.6(\mathrm{Ar}-\mathrm{CH}), 129.2$ (2 x Ar- $\mathrm{CH})$, $129.4(4 \mathrm{x} \mathrm{Ar}-\mathrm{CH}), 130.0(\mathrm{Ar}-\mathrm{CH}), 131.9(\mathrm{Ar}-C), 132.2(\mathrm{Ar}-C), 135.0(\mathrm{Ar}-C), 138.1$ (Ar-C), $142.3(\mathrm{Ar}-\mathrm{C}), 164.9(C=\mathrm{O})$; HRMS $\left(\mathrm{FAB}^{+}\right)$Found: $\left[\mathrm{M}+\mathrm{H}^{+}\right]$288.1388. $\mathrm{C}_{20} \mathrm{H}_{18} \mathrm{NO}$ requires 288.1388; $\mathrm{m} / \mathrm{z}\left(\mathrm{FAB}^{+}\right) 288\left(\left[\mathrm{M}+\mathrm{H}^{+}\right], 75 \%\right), 119(100), 102(44)$.

$\mathrm{N}$-Biphenyl-2-yl-4-tert-butylbenzamide (3c). 4-tert-Butylbenzoyl chloride (2.16 $\mathrm{ml}, 11.82$ mmol) yielded $N$-biphenyl-2-yl-4-(tert-butyl) benzamide 3c $(2.29 \mathrm{~g}, 59 \%)$ as off white crystals mp $160-161^{\circ} \mathrm{C} . v_{\max }(\mathrm{KBr}) / \mathrm{cm}^{-1} 3370,1620 ; \delta_{\mathrm{H}}\left(400 \mathrm{MHz}, \mathrm{CDCl}_{3}\right) 1.31\left[9 \mathrm{H}, \mathrm{s}, \mathrm{C}\left(\mathrm{CH}_{3}\right)_{3}-H\right], 7.21$ $(1 \mathrm{H}, \mathrm{dt}, J 1.2,7.2, \mathrm{Ar}-H), 7.29(1 \mathrm{H}, \mathrm{dd}, J 1.67 .2$, Ar- $H), 7.24(6 \mathrm{H}, \mathrm{m}, \mathrm{Ar}-H), 7.53(4 \mathrm{H}, \mathrm{m}, \mathrm{Ar}-$

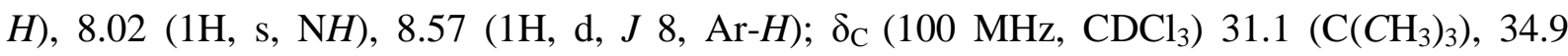
$\left[C\left(\mathrm{CH}_{3}\right)_{3}\right], 121.0(\mathrm{Ar}-\mathrm{CH}), 124.2(\mathrm{Ar}-\mathrm{CH}), 125.7(2 \times \mathrm{Ar}-\mathrm{CH}), 126.7(2 \times \mathrm{Ar}-\mathrm{CH}), 128.2(\mathrm{Ar}-$ $C H), 128.6(\mathrm{Ar}-\mathrm{CH}), 129.2$ (2 x Ar- $\mathrm{CH}), 129.4$ (2 x Ar- $\mathrm{CH}), 130.0(\mathrm{Ar}-\mathrm{CH}), 131.8(\mathrm{Ar}-\mathrm{C}), 132.2$ $(\mathrm{Ar}-C), 135.1(\mathrm{Ar}-C), 138.1(\mathrm{Ar}-C), 155.3(\mathrm{Ar}-C), 164.8(C=\mathrm{O})$; HRMS $\left(\mathrm{FAB}^{+}\right)$Found: $\left[\mathrm{M}+\mathrm{H}^{+}\right]$

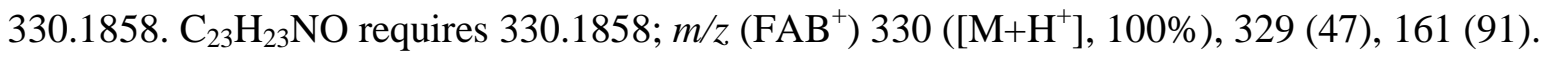

$\mathrm{N}$-Biphenyl-2-yl-4-methoxybenzamide (3d). 4-Methoxybenzoyl chloride (0.40 ml, $2.95 \mathrm{mmol})$ yielded $N$-biphenyl-2-yl-4-methoxybenzamide 3d $(630 \mathrm{mg}, 70 \%)$ as an off white solid mp 131$132{ }^{\circ} \mathrm{C} . \delta_{\mathrm{H}}\left(400 \mathrm{MHz}, \mathrm{CDCl}_{3}\right) 3.82\left(3 \mathrm{H}, \mathrm{s}, \mathrm{CH}_{3}\right) 6.87(2 \mathrm{H}, \mathrm{m}, \mathrm{Ar}-H), 7.20(1 \mathrm{H}, \mathrm{dt}, J$ 1.2, 7.2 , Ar- $H), 7.28(1 \mathrm{H}, \mathrm{m}, \mathrm{Ar}-H), 7.49(8 \mathrm{H}, \mathrm{m}, \mathrm{Ar}-H), 7.93(1 \mathrm{H}, \mathrm{s}, \mathrm{N} H), 8.52(1 \mathrm{H}, \mathrm{m}, \mathrm{Ar}-H) ; \delta_{\mathrm{C}}(100$ $\left.\mathrm{MHz}, \mathrm{CDCl}_{3}\right) 55.4\left(\mathrm{OCH}_{3}\right), 113.9(2 \times \mathrm{Ar}-\mathrm{CH}), 121.0(\mathrm{Ar}-\mathrm{CH}), 124.1(\mathrm{Ar}-\mathrm{CH}), 127.0(\mathrm{Ar}-\mathrm{C})$, 128.1 (Ar- $\mathrm{CH}), 128.6$ (2 x Ar- $\mathrm{CH}), 128.7$ (2 x Ar- $\mathrm{CH}), 129.2$ (2 x Ar- $\mathrm{CH}), 129.4(\mathrm{Ar}-\mathrm{CH}), 129.9$ $(\mathrm{Ar}-\mathrm{CH}), 132.1$ (Ar-C), 135.1 (Ar-C), 138.1 (Ar-C), 162.4 (Ar-COMe), $164.5(C=\mathrm{O})$; HRMS $\left(\mathrm{FAB}^{+}\right)$Found: $\left[\mathrm{M}+\mathrm{H}^{+}\right]$303.1260. $\mathrm{C}_{20} \mathrm{H}_{17} \mathrm{NO}_{2}$ requires 303.1259; $\mathrm{m} / \mathrm{z}\left(\mathrm{FAB}^{+}\right) 304\left(\left[\mathrm{M}+\mathrm{H}^{+}\right]\right.$, 100\%) 303 (39), 135 (9).

$\boldsymbol{N}$-Biphenyl-2-yl-4-chlorobenzamide (3e). 4-Chlorobenzoyl chloride (2.29 ml, $18.02 \mathrm{mmol})$ yielded $N$-biphenyl-2-yl-4-chlorobenzamide $3 \mathrm{e}(3.57 \mathrm{~g}, 64 \%)$ as white crystals mp $104-105{ }^{\circ} \mathrm{C}$. $v_{\max }(\mathrm{KBr}) / \mathrm{cm}^{-1} 3253,1650,1050 ; \delta_{\mathrm{H}}\left(400 \mathrm{MHz}, \mathrm{CDCl}_{3}\right) 7.23(1 \mathrm{H}, \mathrm{dt}, J 1.2,7.6, \mathrm{Ar}-H), 7.31$ $(1 \mathrm{H}, \mathrm{dd}, J$ 1.6, 7.6, Ar- $H), 7.36(2 \mathrm{H}, \mathrm{m}, \mathrm{Ar}-H), 7.44(4 \mathrm{H}, \mathrm{m}, \mathrm{Ar}-H), 7.52(4 \mathrm{H}, \mathrm{m}, \mathrm{Ar}-H), 7.93$ $(1 \mathrm{H}, \mathrm{s}, \mathrm{NH}), 8.49(1 \mathrm{H}, \mathrm{d}, J$ 8.0, $\mathrm{Ar}-H) ; \delta_{\mathrm{C}}\left(100 \mathrm{MHz}, \mathrm{CDCl}_{3}\right) 121.1(\mathrm{Ar}-\mathrm{CH}), 124.6(\mathrm{Ar}-\mathrm{CH})$, $128.2(2 \times \mathrm{Ar}-\mathrm{CH}), 128.3(\mathrm{Ar}-\mathrm{CH}), 128.6(\mathrm{Ar}-\mathrm{CH}), 129.0(2 \times \mathrm{Ar}-\mathrm{CH}), 129.2(2 \mathrm{x} \mathrm{Ar}-\mathrm{CH}), 129.3$ $(2$ x Ar- $C H$ ), $130.0(\mathrm{Ar}-\mathrm{CH}), 132.3(\mathrm{Ar}-\mathrm{C}), 133.1$ (Ar-C), $134.6(\mathrm{Ar}-\mathrm{C}), 137.9(\mathrm{Ar}-\mathrm{C}), 138.0(\mathrm{Ar}-$ $C), 163.9(C=\mathrm{O})$; HRMS $\left(\mathrm{FAB}^{+}\right)$Found: $\left[\mathrm{M}+\mathrm{H}^{+}\right]$308.0841. $\mathrm{C}_{19} \mathrm{H}_{15} \mathrm{NOCl}$ requires 308.0842; $\mathrm{m} / \mathrm{z}$ $\left(\mathrm{FAB}^{+}\right) 308\left(\left[\mathrm{M}+\mathrm{H}^{+}\right], 100 \%\right), 307$ (68), 154 (22), 141 (32), 139 (96).

$\mathrm{N}$-(Biphenyl-2-yl-4-trifluoromethyl)benzamide (3f). 4-Trifluoromethylbenzoyl chloride (1.32 $\mathrm{ml}, 8.86 \mathrm{mmol})$ yielded $N$-(biphenyl-2-yl-4-trifluoro methyl) benzamide $\mathbf{3 f}(2.33 \mathrm{~g}, 77 \%)$ as offwhite crystals mp $127-128^{\circ} \mathrm{C} . v_{\max }(\mathrm{KBr}) / \mathrm{cm}^{-1} 3325,1650 ; \delta_{\mathrm{H}}\left(400 \mathrm{MHz}, \mathrm{CDCl}_{3}\right) 7.25(1 \mathrm{H}, \mathrm{m}$, Ar- $H), 7.33(1 \mathrm{H}, \mathrm{dd}, J$ 1.6, 7.6, Ar- $H), 7.45(4 \mathrm{H}, \mathrm{m}, \mathrm{Ar}-H), 7.53(2 \mathrm{H}, \mathrm{m}, \mathrm{Ar}-H), 7.68(4 \mathrm{H}, \mathrm{dd}, J$ 8.4, $18.8 \mathrm{Ar}-H), 8.00(1 \mathrm{H}, \mathrm{s}, \mathrm{NH}), 8.51(1 \mathrm{H}, \mathrm{d}, J$ 8.4, $\mathrm{Ar}-H) ; \delta_{\mathrm{C}}\left(100 \mathrm{MHz}, \mathrm{CDCl}_{3}\right) 121.2(\mathrm{Ar}-\mathrm{C})$, 
$124.8\left(\mathrm{CF}_{3}\right), 125.8(2 \times \mathrm{Ar}-\mathrm{CH}), 125.9(2 \times \mathrm{Ar}-\mathrm{CH}), 127.3$ (2 x Ar- $\left.\mathrm{CH}\right), 128.4(2 \times \mathrm{Ar}-\mathrm{CH})$, $128.7(2 \times \mathrm{Ar}-\mathrm{CH}), 129.3(2 \times \mathrm{Ar}-\mathrm{CH}), 130.1(\mathrm{Ar}-\mathrm{CH}), 132.5(\mathrm{Ar}-\mathrm{C}), 134.5(\mathrm{Ar}-\mathrm{C}), 137.9(\mathrm{Ar}-$ $C), 138.0(\mathrm{Ar}-C), 163.8(C=\mathrm{O})$; HRMS $\left(\mathrm{FAB}^{+}\right)$Found: $\left[\mathrm{M}+\mathrm{H}^{+}\right]$342.1103. $\mathrm{C}_{20} \mathrm{H}_{14} \mathrm{~F}_{3} \mathrm{NO}$ requires 342.1106); $m / z\left(\mathrm{FAB}^{+}\right) 342\left(\left[\mathrm{M}+\mathrm{H}^{+}\right]\right.$88\%), 341 (57), 173 (100), 145 (44).

$\mathrm{N}$-Biphenyl-2-yl-4-dimethylaminobenzamide (3g). 4-Dimethylaminobenzoyl chloride (1.63 g, $8.86 \mathrm{mmol}$ ) yielded $N$-biphenyl-2-yl-4-dimethylamino benzamide $3 \mathrm{~g}$ (1.72 g, 61\%) as off white crystals mp 204-205 ${ }^{\circ} \mathrm{C} . v_{\max }(\mathrm{KBr}) / \mathrm{cm}^{-1} 3370,1650 ; \delta_{\mathrm{H}}\left(400 \mathrm{MHz}, \mathrm{CDCl}_{3}\right) 2.99[6 \mathrm{H}, \mathrm{s}$, $\left.\mathrm{N}\left(\mathrm{CH}_{3}\right)_{2}\right], 6.60(2 \mathrm{H}, \mathrm{dd}, J 2.0,6.8, \mathrm{Ar}-H), 7.17(1 \mathrm{H}, \mathrm{dt}, J 1.2,7.6, \mathrm{Ar}-H), 7.26(1 \mathrm{H}, \mathrm{dd}, J 1.2,7.6$, $\mathrm{Ar}-H), 7.46(8 \mathrm{H}, \mathrm{m}, \mathrm{Ar}-H), 7.92(1 \mathrm{H}, \mathrm{s}, \mathrm{NH}), 8.57(1 \mathrm{H}, \mathrm{dd}, J 1.2,8.4, \mathrm{Ar}-H) ; \delta_{\mathrm{C}}(100 \mathrm{MHz}$, $\left.\mathrm{CDCl}_{3}\right) 40.1\left(2 \times \mathrm{CH}_{3}\right), 111.1(2 \times \mathrm{Ar}-\mathrm{CH}), 120.8(\mathrm{Ar}-\mathrm{CH}), 121.2(\mathrm{Ar}-\mathrm{C}), 123.6(\mathrm{Ar}-\mathrm{CH}), 128.0$ $(\mathrm{Ar}-\mathrm{CH}), 128.4$ (2 x Ar- $\mathrm{CH}), 128.5(\mathrm{Ar}-\mathrm{CH}), 129.2$ (2 x Ar- $\mathrm{CH}), 129.4$ (2 x Ar-CH), 129.9 (Ar$C H$ ), 131.8 (Ar- $C$ ), 135.5 (Ar- $C$ ), 138.3 (Ar-C), 152.5 (Ar-C), $165.0(C=\mathrm{O})$; HRMS (FAB ${ }^{+}$) Found: $\left[\mathrm{M}+\mathrm{H}^{+}\right]$317.1659. $\mathrm{C}_{21} \mathrm{H}_{21} \mathrm{~N}_{2} \mathrm{O}$ requires $317.1654 ; \mathrm{m} / z\left(\mathrm{FAB}^{+}\right) 317\left(\left[\mathrm{M}+\mathrm{H}^{+}\right], 46 \%\right), 316$ (38), 148 (100).

$\mathrm{N}$-Biphenyl-2-yl-4-nitrobenzamide (3h). 4-Nitrobenzoyl chloride (1.64 g, $8.86 \mathrm{mmol}$ ) yielded $\mathrm{N}$-biphenyl-2-yl-4-nitrobenzamide $3 \mathrm{~h}(1.24 \mathrm{~g}, 44 \%)$ as an off-white solid mp $109-110{ }^{\circ} \mathrm{C} . v_{\max }$ $(\mathrm{KBr}) / \mathrm{cm}^{-1} 1364 ; \delta_{\mathrm{H}}\left(400 \mathrm{MHz}, \mathrm{CDCl}_{3}\right) 7.28(1 \mathrm{H}, \mathrm{m}, \mathrm{Ar}-H), 7.34(2 \mathrm{H}, \mathrm{m}, \mathrm{Ar}-H), 7.50(6 \mathrm{H}, \mathrm{m}$, Ar- $H), 7.75(2 \mathrm{H}, \mathrm{d}, J$ 8.8, Ar- $H), 8.00(1 \mathrm{H}, \mathrm{s}, \mathrm{NH}), 8.24(2 \mathrm{H}, \mathrm{m}, \mathrm{Ar}-H), 8.50(1 \mathrm{H}, \mathrm{d}, J$ 8, Ar- $H)$; $\delta_{\mathrm{C}}\left(100 \mathrm{MHz}, \mathrm{CDCl}_{3}\right)$ 121.2 (Ar-CH), $124.0(2 \mathrm{x} \mathrm{Ar}-\mathrm{CH}), 125.1(\mathrm{Ar}-\mathrm{CH}), 128.0(2 \mathrm{x} \mathrm{Ar}-\mathrm{CH})$, $128.5(\mathrm{Ar}-\mathrm{CH}), 128.9(\mathrm{Ar}-\mathrm{CH}), 129.3$ (2 x Ar- $\mathrm{CH}), 129.4$ (2 x Ar- $\mathrm{CH}), 130.1(\mathrm{Ar}-\mathrm{CH}), 132.7$ $(\operatorname{Ar}-C), 134.2(\operatorname{Ar}-C), 137.7(\operatorname{Ar}-C), 140.3(\mathrm{Ar}-C), 149.7(C=\mathrm{O})$; HRMS (FAB $\left.{ }^{+}\right)$Found: $\left[\mathrm{M}+\mathrm{H}^{+}\right]$ 319.1088. $\mathrm{C}_{19} \mathrm{H}_{14} \mathrm{~N}_{2} \mathrm{O}_{3}$ requires: 319.1083; $\mathrm{m} / z\left(\mathrm{FAB}^{+}\right) 319\left(\left[\mathrm{M}+\mathrm{H}^{+}\right], 29 \%\right), 176(40), 154$ (100), $136(58)$.

\section{General experimental procedures for the preparation of the imidoyl-selanides 2}

To a solution of amide 3 (1.0 equiv.) in dry $\mathrm{CH}_{2} \mathrm{Cl}_{2}(8 \mathrm{ml} / \mathrm{mmol})$ under inert atmosphere was added dry DMF ( 1 drop/mmol) and phosgene (3.0 equiv.). The reaction mixture was stirred at $\mathrm{rt}$ for $5 \mathrm{~h}$. The volatiles were then removed under reduced pressure to give the crude imidoyl chloride as a residue.

K-Selectride ${ }^{\circledR}$ (1M THF solution, 1.1 equiv.) was added to a solution of ( $\left.\mathrm{PhSe}\right)_{2}(0.5$ equiv.) in THF $(10 \mathrm{ml} / \mathrm{mmol})$ to form a white suspension. In a separate flask, the crude imidoyl chloride was re-dissolved in dry THF $(8 \mathrm{ml} / \mathrm{mmol})$ and the solution was cannulated into the suspension. The reaction mixture was stirred at rt overnight. The solvent was then removed under reduced pressure and the residue was partitioned between water $(8 \mathrm{ml} / \mathrm{mmol})$ and $\mathrm{CH}_{2} \mathrm{Cl}_{2}(8 \mathrm{ml} / \mathrm{mmol})$. The organics were separated, dried over $\mathrm{MgSO}_{4}$, filtered and concentrated under reduced pressure to give crude imidoyl-selanide product 2 . No further purification was carried out in most cases.

Phenyl- $\boldsymbol{N}$-biphenyl-2-ylbenzimidoselenoate (2a). Amide 3a (1.39 g, 5.09 mmol) yielded phenyl- $N$-biphenyl-2-ylbenzimidoselenoate $2 \mathbf{a}$ (1.74 g crude) and it was carried on to the next reaction without further purification. 
$N$-Biphenyl-2-yl-4-methylselenobenzimidic acid phenyl ester (2b). Amide 3b (1.00 g, 3.48 mmol) yielded $N$-biphenyl-2-yl-4-methylselenobenzimidic acid phenyl ester $\mathbf{2 b}$ (655 mg crude) as a pale yellow solid mp $104-105{ }^{\circ} \mathrm{C} . v_{\max }(\mathrm{KBr}) / \mathrm{cm}^{-1} 1627,1535 ; \delta_{\mathrm{H}}\left(400 \mathrm{MHz}, \mathrm{CDCl}_{3}\right) 2.21$ $\left(3 \mathrm{H}, \mathrm{s}, \mathrm{CH}_{3}\right), 7.22(18 \mathrm{H}, \mathrm{m}, \mathrm{Ar}-\mathrm{H}) ; \delta_{\mathrm{C}}\left(100 \mathrm{MHz}, \mathrm{CDCl}_{3}\right) 21.3\left(\mathrm{CH}_{3}\right), 120.1(\mathrm{Ar}-\mathrm{CH}), 125.1(2 \mathrm{x}$ $\mathrm{Ar}-\mathrm{CH}), 126.9(\mathrm{Ar}-\mathrm{CH}), 127.4(2 \times \mathrm{Ar}-\mathrm{CH}), 127.9(2 \times \mathrm{Ar}-\mathrm{CH}), 128.0(2 \times \mathrm{Ar}-\mathrm{CH}), 128.4(2 \times$ $\mathrm{Ar}-\mathrm{CH}), 128.7$ ( $2 \times \mathrm{Ar}-\mathrm{CH}), 129.1(\mathrm{Ar}-\mathrm{CH}), 129.7$ (Ar-C), $129.8(2 \times \mathrm{Ar}-\mathrm{CH}), 130.4$ (Ar- $\mathrm{CH})$, 131.8 (Ar-C), $134.9(\mathrm{Ar}-\mathrm{CH}), 135.3(\mathrm{Ar}-\mathrm{C}), 139.7(\mathrm{Ar}-C), 140.0(\mathrm{Ar}-C), 149.1(C=\mathrm{N})$; HRMS $\left(\mathrm{FAB}^{+}\right)$Found: $\left[\mathrm{M}+\mathrm{H}^{+}\right]$428.0916. $\mathrm{C}_{26} \mathrm{H}_{22} \mathrm{NSe}$ requires 428.0917; $\mathrm{m} / \mathrm{z}\left(\mathrm{FAB}^{+}\right) 428\left(\left[\mathrm{M}+\mathrm{H}^{+}\right]\right.$, 67\%), 427 (100), 91 (56).

$N$-Biphenyl-2-yl-4-tert-butyl selenobenzimidic acid phenyl ester (2c). Amide 3c (1.00 g, 3.04 mmol) yielded $N$-biphenyl-2-yl-4-tert-butyl selenobenzimidic acid phenyl ester 2c (1.53 g, crude) and it was carried on to the next reaction without further purification.

$N$-Biphenyl-2-yl-4-methoxyselenobenzimidic acid phenyl ester (2d). Amide 3d (2.10 g, 6.63 mmol) yielded $N$-biphenyl-2-yl-4-methoxyselenobenzimidic acid phenyl ester 2d (51\% crude) and it was carried on to the next reaction without further purification.

$N$-Biphenyl-2-yl-4-chloroselenobenzimidic acid phenyl ester (2e). Amide 3e (1.00 g, 3.25 mmol) yielded $N$-biphenyl-2-yl-4-chloroselenobenzimidic acid phenyl ester $2 \mathbf{e}(659 \mathrm{mg}, 60 \%)$ as yellow crystals mp $116-117{ }^{\circ} \mathrm{C}$. It was carried on to the next reaction without further purification. Decomposition occurred before spectra could be recorded.

$\boldsymbol{N}$-(Biphenyl-2-yl-4-trifloromethyl)selenobenzimidic acid phenyl ester (2f). Amide $3 \mathbf{f}$ ( $2.26 \mathrm{~g}$, $6.63 \mathrm{mmol}$ ) yielded $N$-(biphenyl-2-yl-4-trifloromethyl) selenobenzimidic acid phenyl ester $\mathbf{2 f}$ $(1.91 \mathrm{~g}, 60 \%)$ and it was isolated as pale yellow crystals mp $100-101{ }^{\circ} \mathrm{C} . v_{\max }(\mathrm{KBr}) / \mathrm{cm}^{-1} 1627$, $1573 ; \delta_{\mathrm{H}}\left(400 \mathrm{MHz}, \mathrm{CDCl}_{3}\right) 7.01(6 \mathrm{H}, \mathrm{m}, \mathrm{Ar}-H), 7.32(10 \mathrm{H}, \mathrm{m}, \mathrm{Ar}-H), 7.56(2 \mathrm{H}, \mathrm{d}, J 7.2, \mathrm{Ar}-H)$; $\delta_{\mathrm{C}}\left(100 \mathrm{MHz}, \mathrm{CDCl}_{3}\right) 119.6(\mathrm{Ar}-\mathrm{CH}), 124.5(\mathrm{Ar}-\mathrm{CH}), 124.6(\mathrm{Ar}-\mathrm{CH}), 125.1(\mathrm{Ar}-\mathrm{C}), 125.5(\mathrm{Ar}-$ $C H), 127.1(\mathrm{Ar}-\mathrm{CH}), 127.9$ (2 x Ar- $\mathrm{CH}), 128.1(\mathrm{Ar}-\mathrm{CH}), 128.2(\mathrm{Ar}-\mathrm{CH}), 128.5(\mathrm{Ar}-\mathrm{C}), 128.9$ (2 x Ar- $\mathrm{CH}), 129.1(\mathrm{Ar}-\mathrm{CH}), 129.6(\mathrm{Ar}-\mathrm{CH}), 129.8(2 \mathrm{x} \mathrm{Ar}-\mathrm{CH}), 130.6(\mathrm{Ar}-\mathrm{CH}), 131.0(\mathrm{Ar}-\mathrm{CH})$, 131.3 (Ar-C), 131.8 (Ar-C), 135.5 (Ar- $C H$ ), 139.5 (Ar-C), 141.3 (Ar-C), 148.7 (Ar-C), 163.5 $(C=\mathrm{N})$; HRMS $\left(\mathrm{FAB}^{+}\right)$Found: $\left[\mathrm{M}+\mathrm{H}^{+}\right]$482.0638. $\mathrm{C}_{26} \mathrm{H}_{18} \mathrm{~F}_{3} \mathrm{~N}^{80} \mathrm{Se}$ requires 482.0635$) ; \mathrm{m} / \mathrm{z}$ $\left(\mathrm{FAB}^{+}\right) 482\left(\left[\mathrm{M}+\mathrm{H}^{+}\right]{ }^{80} \mathrm{Se}, 13 \%\right), 480\left({ }^{78} \mathrm{Se}, 7\right), 325$ (70), 324 (100), 178 (24), 152 (52).

Phenyl- $N$-biphenyl-2-yl-4-(dimethylamino)benzimidoselenoate (2g). Amide 3g (1.50 g, 4.74 mmol) yielded phenyl- $N$-biphenyl-2-yl-4-(dimethylamino) benzimidoselenoate $2 \mathrm{~g}$ (282 $\mathrm{mg}, 62 \%$ crude) and it was carried on to the next reaction without further purification.

\section{General experimental procedure for the preparation of imine 5}

To a solution of 2-aminobiphenyl 4 (1.0 equiv.) in $\mathrm{CH}_{2} \mathrm{Cl}_{2}(2 \mathrm{ml} / \mathrm{mmol})$ in the presence of $4 \AA$ molecular sieves was added aldehyde (1.0 equiv.). The reaction mixture was stirred at $\mathrm{rt}$ for $5 \mathrm{~h}$. It was then filtered through a pad of Celite and washed with excess DCM. The filtrate was concentrated under reduced pressure to give imine product 5 without further purification. 
Benzylidene biphenyl-2-yl amine (5a). ${ }^{7}$ Benzaldehyde $(0.30 \mathrm{ml}, 2.95 \mathrm{mmol})$ yielded benzylidene biphenyl-2-yl amine 5a (455 mg, 60\%) as a yellow oil. The crude imine 5a was directly carried on to the next reaction without isolation.

Biphenyl-2-yl-(4-methylbenzylidene) amine (5b). $p$-Tolualdehyde $(0.35 \mathrm{ml}, 2.95 \mathrm{mmol})$ yielded biphenyl-2-yl-(4-methylbenzylidene) amine $\mathbf{5 b}(592 \mathrm{mg}, 66 \%)$ as yellow crystals $\mathrm{mp}$ 79$80{ }^{\circ} \mathrm{C} . v_{\max }(\mathrm{KBr}) / \mathrm{cm}^{-1} 2260 ; \delta_{\mathrm{H}}\left(400 \mathrm{MHz}, \mathrm{CDCl}_{3}\right) 2.37\left(3 \mathrm{H}, \mathrm{s}, \mathrm{CH}_{3}\right), 7.06(1 \mathrm{H}, \mathrm{dd}, J$ 0.12, 7.6, Ar-H), $7.21(2 \mathrm{H}, \mathrm{d}, J$ 7.6, Ar- $H), 7.47(3 \mathrm{H}, \mathrm{m}, \mathrm{Ar}-H), 7.68(2 \mathrm{H}, \mathrm{d}, J$ 8.4, Ar- $H), 8.41(1 \mathrm{H}, \mathrm{s}$, imine- $H) ; \delta_{\mathrm{C}}\left(100 \mathrm{MHz}, \mathrm{CDCl}_{3}\right) 21.6\left(\mathrm{CH}_{3}\right), 119.0(\mathrm{Ar}-\mathrm{CH}), 125.8(\mathrm{Ar}-\mathrm{CH}), 126.7(\mathrm{Ar}-\mathrm{CH})$, 127.6 ( 2 x Ar-CH), 128.3 (Ar-CH), 128.8 ( 2 x Ar-CH), 129.4 ( 2 x Ar- $\mathrm{CH}), 130.2$ ( 2 x Ar- $\mathrm{CH})$, 130.3 (Ar- $C H$ ), 133.9 (Ar-C), 135.2 (Ar-C), 139.5 (Ar-C), 141.7 (Ar-C), 149.9 (Ar-C), 160.2 (imine-C); HRMS $\left(\mathrm{FAB}^{+}\right)$Found: $\left[\mathrm{M}+\mathrm{H}^{+}\right] 272.1439 . \mathrm{C}_{20} \mathrm{H}_{18} \mathrm{~N}$ requires $272.1439 ; \mathrm{m} / \mathrm{z}\left(\mathrm{FAB}^{+}\right)$ $272\left(\left[\mathrm{M}+\mathrm{H}^{+}\right], 100 \%\right), 271$ (55), 270 (31), 180 (56).

Biphenyl-2-yl-(4-tert-butylbenzylidene) amine (5c). 4-t-Butylbenzaldehyde (0.49 ml, 2.95 mmol) yielded biphenyl-2-yl-(4-t-butylbenzylidene) amine 5c $(770 \mathrm{mg}, 86 \%)$ as a yellow oil. $v_{\max }($ neat $) / \mathrm{cm}^{-1} 1630,1540 ; \delta_{\mathrm{H}}\left(400 \mathrm{MHz}, \mathrm{CDCl}_{3}\right) 1.34\left(9 \mathrm{H}, \mathrm{s},{ }^{\mathrm{t}} \mathrm{Bu}-H\right), 7.06(1 \mathrm{H}, \mathrm{dd}, J$ 1.2, 8, Ar-H), 7.29 (2H, m, Ar-H), $7.36(3 \mathrm{H}, \mathrm{m}, \mathrm{Ar}-H), 7.47(5 \mathrm{H}, \mathrm{m}, \mathrm{Ar}-H), 7.73(2 \mathrm{H}, \mathrm{d}, J$ 8.4, Ar- $H$ ), 8.44 (imine- $\left.H) ; \delta_{\mathrm{C}}\left(100 \mathrm{MHz}, \mathrm{CDCl}_{3}\right) 31.2\left(\mathrm{C}_{(\mathrm{CH}}\right)_{3}\right), 35.0\left(\mathrm{C}\left(\mathrm{CH}_{3}\right)_{3}\right), 119.0(\mathrm{Ar}-\mathrm{CH}), 125.7$ (2 x $\mathrm{Ar}-\mathrm{CH}), 125.8(\mathrm{Ar}-\mathrm{CH}), 126.0(\mathrm{Ar}-\mathrm{CH}), 127.7(2 \times \mathrm{Ar}-\mathrm{CH}), 128.3(\mathrm{Ar}-\mathrm{CH}), 128.7$ (2 x Ar$C H$ ), 130.2 (2 x Ar- $C H$ ), $130.3(\mathrm{Ar}-\mathrm{CH}), 133.9$ (Ar-C), 135.3 (Ar-C), 139.5 (Ar-C), 149.9 (Ar$C$ ), 154.7 (Ar-C), 160.1 (imine-C); HRMS (FAB ${ }^{+}$) Found: $\left[\mathrm{M}+\mathrm{H}^{+}\right] 314.1911 . \mathrm{C}_{23} \mathrm{H}_{24} \mathrm{~N}$ requires 314.1909; $m / z\left(\mathrm{FAB}^{+}\right) 314\left(\left[\mathrm{M}+\mathrm{H}^{+}\right], 64 \%\right), 169$ (44), 148 (100), 57 (20).

Biphenyl-2-yl-(4-methoxybenzylidene) amine (5d). ${ }^{7}$ 4-Methoxybenzaldehyde (0.36 ml, 2.95 mmol) yielded biphenyl-2-yl-(4-methoxybenzylidene) amine 5d (661 mg, 78\%) as a yellow oil. The crude imine $\mathbf{5 d}$ was directly carried on to the next reaction without isolation.

Biphenyl-2-yl-(4-chlorobenzylidene) amine (5e). ${ }^{7}$ 4-Chlorobenzaldehyde (378 mg, 2.69 mmol) yielded biphenyl-2-yl-(4-chlorobenzylidene) amine 5e (619 mg, 79\%) as yellow crystals mp 83$84{ }^{\circ} \mathrm{C}$ (lit. $\left.{ }^{7} 89-90^{\circ} \mathrm{C}\right) . v_{\max }$ (thin film)/ $\mathrm{cm}^{-1} 1627,1087 ; \delta_{\mathrm{H}}\left(400 \mathrm{MHz}, \mathrm{CDCl}_{3}\right) 7.06(1 \mathrm{H}, \mathrm{dd}, J 1.2$, 7.6, Ar- $H), 7.38(10 \mathrm{H}, \mathrm{m}, \mathrm{Ar}-H), 7.70(2 \mathrm{H}, \mathrm{dt}, J 2,8.8, \operatorname{Ar}-H), 8.40(1 \mathrm{H}, \mathrm{s}$, imine- $H) ; \delta_{\mathrm{C}}(100$ $\left.\mathrm{MHz}, \mathrm{CDCl}_{3}\right) 118.7$ (Ar-C), $126.2(\mathrm{Ar}-\mathrm{CH}), 126.8(\mathrm{Ar}-\mathrm{CH}), 127.7$ (2 Ar- $\left.\mathrm{CH}\right), 128.4(\mathrm{Ar}-\mathrm{CH})$, 129.0 (2 Ar- $\mathrm{CH}), 129.9$ (2 Ar- $\mathrm{CH}), 130.2$ (2 Ar- $\mathrm{CH}), 130.4(\mathrm{Ar}-\mathrm{CH}), 134.8(\mathrm{Ar}-\mathrm{C}), 135.4$ (Ar$C), 137.2(\mathrm{Ar}-C), 139.4(\mathrm{Ar}-C), 149.2\left(2^{\prime}-C\right), 158.8(7-C)$; HRMS (FAB $\left.{ }^{+}\right)$Found: $\left[\mathrm{M}+\mathrm{H}^{+}\right]$ 292.0897. $\mathrm{C}_{19} \mathrm{H}_{15} \mathrm{NCl}^{35}$ requires 292.0893; $\mathrm{m} / z\left(\mathrm{FAB}^{+}\right) 292\left(\left[\mathrm{M}+\mathrm{H}^{+}\right], 70 \%\right), 291$ (32), 290 (31), 180 (100), 152 (26).

Biphenyl-2-yl-(4-trifluoromethylbenzylidene) amine (5f). 4-Trifluoromethylbenzaldehyde $(0.40 \mathrm{ml}, 2.95 \mathrm{mmol})$ yielded biphenyl-2-yl-(4-trifluoromethyl benzylidene) amine $\mathbf{5 f}$ (561 $\mathrm{mg}$, $58 \%)$ as yellow crystals mp $97-98{ }^{\circ} \mathrm{C} . v_{\max }(\mathrm{KBr}) / \mathrm{cm}^{-1} 1630 ; \delta_{\mathrm{H}}\left(400 \mathrm{MHz}, \mathrm{CDCl}_{3}\right) 7.09(1 \mathrm{H}, \mathrm{dd}$, $J$ 1.6 8.0, Ar-H), 7.32 (5H, m, Ar- $H), 7.47(3 \mathrm{H}, \mathrm{m}, \mathrm{Ar}-H), 7.66(2 \mathrm{H}, \mathrm{d}, J$ 8.4, Ar- $H), 7.88(2 \mathrm{H}, \mathrm{d}$, $J$ 8.0, Ar- $H), 8.49(1 \mathrm{H}, \mathrm{s}$, imine- $H) ; \delta_{\mathrm{C}}\left(100 \mathrm{MHz}, \mathrm{CDCl}_{3}\right) 118.6(\mathrm{Ar}-\mathrm{CH}), 125.5\left(\mathrm{CF}_{3}\right), 125.6$ $(\mathrm{Ar}-\mathrm{CH}), 125.7(\mathrm{Ar}-\mathrm{CH}), 125.8(\mathrm{Ar}-\mathrm{C}), 126.6(\mathrm{Ar}-\mathrm{CH}), 126.9(\mathrm{Ar}-\mathrm{CH}), 127.8(2 \times \mathrm{Ar}-\mathrm{CH})$, $128.4(\mathrm{Ar}-\mathrm{CH}), 129.0(2 \times \mathrm{Ar}-\mathrm{CH}), 130.2$ (2 x Ar- $\mathrm{CH}), 130.5$ (Ar- $\mathrm{CH}), 135.6(\mathrm{Ar}-\mathrm{C}), 139.3$ (Ar- 
C), $139.4(\mathrm{Ar}-C), 149.0(\mathrm{Ar}-C), 158.6$ (imine-C); HRMS (FAB ${ }^{+}$) Found: $\left[\mathrm{M}+\mathrm{H}^{+}\right] 326.1157$. $\mathrm{C}_{20} \mathrm{H}_{14} \mathrm{~F}_{3} \mathrm{~N}$ requires 326.1157); $m / z\left(\mathrm{FAB}^{+}\right) 326\left(\left[\mathrm{M}+\mathrm{H}^{+}\right], 100 \%\right), 325$ (67), 324 (39), 180 (92), 152 (26).

Biphenyl-2-yl-(4-dimethylaminobenzylidene) amine (5g). 4-Dimethylaminobenzaldehyde (440 mg, $2.95 \mathrm{mmol}$ ) yielded biphenyl-2-yl-(4-dimethylamino benzylidene) amine $\mathbf{5 g}$ (588 $\mathrm{mg}$, $66 \%)$ as a yellow crystals mp $82-83{ }^{\circ} \mathrm{C} . v_{\max }(\mathrm{KBr}) / \mathrm{cm}^{-1} 1690 ; \delta_{\mathrm{H}}\left(400 \mathrm{MHz}, \mathrm{CDCl}_{3}\right) 3.00(6 \mathrm{H}$, s, $\left.\mathrm{N}\left(\mathrm{CH}_{3}\right)_{2}\right), 6.68(2 \mathrm{H}, \mathrm{dd}, J 1.6,6.8, \mathrm{Ar}-H), 7.06(1 \mathrm{H}, \mathrm{dd}, J 1.2,7.6, \mathrm{Ar}-H), 7.25(2 \mathrm{H}, \mathrm{m}, \mathrm{Ar}-H)$, 7.34 (3H, m, Ar-H), 7.44 (1H, dd, J 1.2, 7.2, Ar-H), 7.51 (2H, m, Ar-H), 7.67 (2H, dd, J 2.0, 7.2, $\operatorname{Ar}-H), 8.32(1 \mathrm{H}, \mathrm{s}$, imine- $H) ; \delta_{\mathrm{C}}\left(100 \mathrm{MHz}, \mathrm{CDCl}_{3}\right) 40.1\left(2 \times \mathrm{CH}_{3}\right), 111.5(2 \times \mathrm{Ar}-\mathrm{CH}), 119.2$ $(\mathrm{Ar}-\mathrm{CH}), 124.8$ (Ar-C), 125.1 (Ar- $\mathrm{CH}), 126.5(\mathrm{Ar}-\mathrm{CH}), 127.6$ (2 x Ar- $\mathrm{CH}), 128.3$ (Ar-CH), 130.2 (Ar- $C H$ ), 130.3 (2 x Ar- $C H$ ), 130.4 (2 x Ar- $C H$ ), 135.1 (Ar-C), 139.8 (Ar-C), 150.6 (Ar$C$ ), 152.4 (Ar-C), 160.0 (imine-C); HRMS (FAB ${ }^{+}$) Found: $\left[\mathrm{M}+\mathrm{H}^{+}\right] 301.1704 . \mathrm{C}_{21} \mathrm{H}_{21} \mathrm{~N}_{2}$ requires 301.1705; $\mathrm{m} / \mathrm{z}\left(\mathrm{FAB}^{+}\right) 301\left(\left[\mathrm{M}+\mathrm{H}^{+}\right], 100 \%\right), 300$ (88), 299 (61), 180 (66).

Biphenyl-2-yl-(4-nitrobenzylidene) amine (5h). ${ }^{7}$ 4-Nitrobenzaldehyde (446 mg, $\left.2.95 \mathrm{mmol}\right)$ yielded biphenyl-2-yl-(4-nitrobenzylidene) amine $\mathbf{5 h}(770 \mathrm{mg}, 86 \%)$ as yellow crystals $\mathrm{mp}$ 105$106{ }^{\circ} \mathrm{C}$ (lit. $\left.{ }^{7} 106-107^{\circ} \mathrm{C}\right) . \delta_{\mathrm{H}}\left(400 \mathrm{MHz}, \mathrm{CDCl}_{3}\right) 7.13(1 \mathrm{H}, \mathrm{dd}, J 1.2,7.6, \mathrm{Ar}-H), 7.39(7 \mathrm{H}, \mathrm{m}, \mathrm{Ar}-$ $H), 7.50(1 \mathrm{H}, \mathrm{dd}, J$ 2.0, 7.2, Ar-H), $7.95(2 \mathrm{H}, \mathrm{dt}, J 2,88, \operatorname{Ar}-H), 8.28(2 \mathrm{H}, \mathrm{dt}, J 2,8.8, \operatorname{Ar}-H)$, $8.56(1 \mathrm{H}, \mathrm{s}$, imine- $H) ; \delta_{\mathrm{C}}\left(100 \mathrm{MHz}, \mathrm{CDCl}_{3}\right) 118.4(\mathrm{Ar}-\mathrm{CH}), 124.0(2 \mathrm{x} \mathrm{Ar}-\mathrm{CH}), 127.0(\mathrm{Ar}-\mathrm{CH})$, 127.1 (Ar- $\mathrm{CH}), 127.8(2 \times \mathrm{Ar}-\mathrm{CH}), 128.5(\mathrm{Ar}-\mathrm{CH}), 129.4(2 \times \mathrm{Ar}-\mathrm{CH}), 130.2(2 \mathrm{x} \mathrm{Ar}-\mathrm{CH}), 130.6$ $(\mathrm{Ar}-\mathrm{CH}), 136.0(\mathrm{Ar}-\mathrm{C}), 139.2(\mathrm{Ar}-C), 141.7(\mathrm{Ar}-C), 148.5(\mathrm{Ar}-C), 149.2$ (Ar- $C$ ), 157.5 (imineC); HRMS (FAB ${ }^{+}$) Found: $\left[\mathrm{M}^{+}\right]$303.1136. $\mathrm{C}_{19} \mathrm{H}_{14} \mathrm{~N}_{2} \mathrm{O}_{2}$ requires 303.1134; $\mathrm{m} / z\left(\mathrm{FAB}^{+}\right) 303\left(\mathrm{M}^{+}\right.$, 100\%), 302 (63), 180 (65), 154 (47), 136 (30).

\section{General experimental procedure for the preparation of phenanthridine 1 by Pd catalysis}

To a solution of imidoyl-selanide 2 (1.0 equiv.) in toluene $(5 \mathrm{ml} / \mathrm{mmol})$ under inert atmosphere was added $\mathrm{Pd}\left(\mathrm{PPh}_{3}\right)_{4}$ (0.4 equiv.) and triethylamine (5.0 equiv.). The reaction mixture was refluxed for $48 \mathrm{~h}$. The solvent was then removed under reduced pressure. The residue was dissolved in DCM $(10 \mathrm{ml} / \mathrm{mmol})$, washed with water, sat. $\mathrm{NaHCO}_{3}$ solution and brine. The organics were dried over $\mathrm{MgSO}_{4}$, filtered and concentrated under reduced pressure. The crude product was purified by column chromatography to yield phenanthridine product $\mathbf{1}$.

6-Phenylphenanthridine (1a). ${ }^{7}$ Imidoyl-selanide 2a (400 mg, $0.97 \mathrm{mmol}$ ) yielded 6-phenyl phenanthridine 1a (59 mg, 24\%) after column chromatography (Alumina, 25\% DCM in hexanes) as white crystals mp $101-102^{\circ} \mathrm{C}$ (lit. $\left.{ }^{7} 103-105{ }^{\circ} \mathrm{C}\right) . v_{\max }(\mathrm{KBr}) / \mathrm{cm}^{-1} 2400 ; \delta_{\mathrm{H}}\left(400 \mathrm{MHz}, \mathrm{CDCl}_{3}\right)$ $7.66(8 \mathrm{H}, \mathrm{m}, \mathrm{Ar}-H), 7.88(1 \mathrm{H}$, dt $J 1.2,7.2$, Ar- $H), 8.12(1 \mathrm{H}, \mathrm{dd}, J 0.4,8.4, \mathrm{Ar}-H), 8.25(1 \mathrm{H}$, dd, $J$ 1.6, 8.4, Ar- $H), 8.64(1 \mathrm{H}, \mathrm{dd}, J 1.2,8.0, \mathrm{Ar}-H), 8.72(1 \mathrm{H}, \mathrm{d}, J$ 8.4, Ar- $H)$; $\delta_{\mathrm{C}}(100 \mathrm{MHz}$, $\left.\mathrm{CDCl}_{3}\right) 122.0$ (2 x Ar- $\left.\mathrm{CH}\right), 122.2$ (2 x Ar- $\left.\mathrm{CH}\right), 123.8$ (Ar-C), 125.2 (Ar-C), 127.0 (Ar- $\mathrm{H}$ ), $127.2(\mathrm{Ar}-\mathrm{CH}), 128.5(2 \times \mathrm{Ar}-\mathrm{CH}), 128.8(\mathrm{Ar}-\mathrm{CH}), 128.9(\mathrm{Ar}-\mathrm{CH}), 129.0(\mathrm{Ar}-\mathrm{CH}), 129.8(2 \times$ $\mathrm{Ar}-\mathrm{CH}), 130.7(\mathrm{Ar}-\mathrm{CH}), 133.5(\mathrm{Ar}-\mathrm{C}), 137.0(\mathrm{Ar}-\mathrm{C}), 161.3(\mathrm{Ar}-\mathrm{C}=\mathrm{N})$; HRMS $\left(\mathrm{FAB}^{+}\right)$Found: $\left[\mathrm{M}^{+}\right]$255.1049. $\mathrm{C}_{19} \mathrm{H}_{13} \mathrm{~N}$ requires 255.1048; $\mathrm{m} / z\left(\mathrm{FAB}^{+}\right) 255\left(\left[\mathrm{M}^{+}\right], 87 \%\right), 77$ (100). 
6-(4-Tolyl)phenanthridine (1b). ${ }^{\mathbf{2 b}}$ Imidoyl-selanide $\mathbf{2 b}(250 \mathrm{mg}, 0.59 \mathrm{mmol})$ yielded 6-(4tolyl)phenanthridine 1b (60 mg, 38\%) after column chromatography (alumina, 25\% DCM in hexanes) as white crystals mp 107-109 ${ }^{\circ} \mathrm{C}\left(\right.$ lit. $\left.^{20} 107.5-108{ }^{\circ} \mathrm{C}\right) . v_{\max }(\mathrm{KBr}) / \mathrm{cm}^{-1} 2925,1610 ; \delta_{\mathrm{H}}$ $\left(400 \mathrm{MHz}, \mathrm{CDCl}_{3}\right) 2.48\left(3 \mathrm{H}, \mathrm{s}, \mathrm{CH}_{3}\right), 7.37(2 \mathrm{H}, \mathrm{m}, \mathrm{Ar}-\mathrm{H}), 7.69(5 \mathrm{H}, \mathrm{m}, \mathrm{Ar}-H), 7.86(1 \mathrm{H}, \mathrm{dt}, J$ 1.2, 7.2, Ar- $H), 8.14(1 \mathrm{H}, \mathrm{m}, \mathrm{Ar}-\mathrm{H}), 8.24(1 \mathrm{H}, \mathrm{dd}, J 1.2,7.6, \mathrm{Ar}-H), 8.62(1 \mathrm{H}, \mathrm{dd}, J 1.6,8.4$, Ar$H), 8.71(1 \mathrm{H}, \mathrm{d}, J$ 8.0, $\mathrm{Ar}-H) ; \delta_{\mathrm{C}}\left(100 \mathrm{MHz}, \mathrm{CDCl}_{3}\right) 21.4\left(\mathrm{CH}_{3}\right), 121.9(\mathrm{Ar}-\mathrm{CH}), 122.2(\mathrm{Ar}-\mathrm{CH})$, $123.7(\mathrm{Ar}-\mathrm{C}), 125.3(\mathrm{Ar}-\mathrm{C}), 126.8(\mathrm{Ar}-\mathrm{CH}), 127.1(\mathrm{Ar}-\mathrm{CH}), 128.8(\mathrm{Ar}-\mathrm{CH}), 129.0(\mathrm{Ar}-\mathrm{CH})$, $129.1(2 \times \mathrm{Ar}-\mathrm{CH}), 129.7$ ( 2 x Ar- $\mathrm{CH}), 130.3(\mathrm{Ar}-\mathrm{CH}), 130.5(\mathrm{Ar}-\mathrm{CH}), 133.5(\mathrm{Ar}-\mathrm{C}), 136.9$ (Ar$C), 138.6(\mathrm{Ar}-C), 143.9(\mathrm{Ar}-C), 161.3(\mathrm{Ar}-C=\mathrm{N})$; HRMS $\left(\mathrm{FAB}^{+}\right)$Found: $\left[\mathrm{M}+\mathrm{H}^{+}\right] 270.1280$. $\mathrm{C}_{20} \mathrm{H}_{16} \mathrm{~N}$ requires $270.1283 ; \mathrm{m} / z\left(\mathrm{FAB}^{+}\right) 270\left(\left[\mathrm{M}+\mathrm{H}^{+}\right], 88 \%\right), 180$ (100).

6-(4-tert-butylphenyl)phenanthridine (1c). Imidoyl-selanide 2c (600 $\mathrm{mg}, 1.28 \mathrm{mmol})$ yielded 6-(4-tert-butylphenyl) phenanthridine 1c $(187 \mathrm{mg}, 47 \%)$ after column chromatography (alumina, $25 \%$ DCM in hexanes) as a clear oil. $v_{\max }(\mathrm{KBr}) / \mathrm{cm}^{-1} 1560 ; \delta_{\mathrm{H}}\left(400 \mathrm{MHz}, \mathrm{CDCl}_{3}\right) 1.41(9 \mathrm{H}, \mathrm{s}$, $\left.\mathrm{C}\left(\mathrm{CH}_{3}\right)_{3}\right), 7.63(6 \mathrm{H}, \mathrm{m}, \mathrm{Ar}-H), 7.75(1 \mathrm{H}, \mathrm{m}, \mathrm{Ar}-H), 7.85(1 \mathrm{H}, \mathrm{m}, \mathrm{Ar}-H), 8.19(1 \mathrm{H}, \mathrm{dd}, J$ 0.8, 8.0, $\operatorname{Ar}-H), 8.27(1 \mathrm{H}, \mathrm{d}, J$ 8.0, Ar- $H), 8.61(1 \mathrm{H}, \mathrm{dd}, J 1.2,8.0, \mathrm{Ar}-H), 8.70(1 \mathrm{H}, \mathrm{d}, J 8.0, \operatorname{Ar}-H) ; \delta_{\mathrm{C}}$

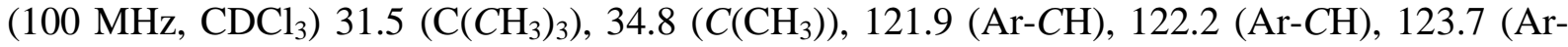
$C), 125.2(\mathrm{Ar}-\mathrm{C}), 125.4(2 \mathrm{x} \mathrm{Ar}-\mathrm{CH}), 126.7(\mathrm{Ar}-\mathrm{CH}), 126.8(\mathrm{Ar}-\mathrm{CH}), 128.8(\mathrm{Ar}-\mathrm{CH}), 129.1(\mathrm{Ar}-$ $C H), 129.5(2 \times \mathrm{Ar}-\mathrm{CH}), 130.2(\mathrm{Ar}-\mathrm{CH}), 130.6(\mathrm{Ar}-\mathrm{CH}), 133.5(\mathrm{Ar}-\mathrm{C}), 136.7(\mathrm{Ar}-\mathrm{C}), 143.7(\mathrm{Ar}-$ $C), 151.8(\mathrm{Ar}-C), 161.3(C=\mathrm{N})$; HRMS $\left(\mathrm{FAB}^{+}\right)$Found: $\left[\mathrm{M}+\mathrm{H}^{+}\right] 312.1753 . \mathrm{C}_{23} \mathrm{H}_{22} \mathrm{~N}$ requires $312.1752 ; m / z\left(\mathrm{FAB}^{+}\right) 312\left(\left[\mathrm{M}+\mathrm{H}^{+}\right], 100 \%\right), 180(35) 134(50)$.

6-(4-Methoxyphenyl)phenanthridine (1d) ${ }^{7}$ Imidoyl-selanide $2 d$ (500 $\mathrm{mg}, 1.13 \mathrm{mmol}$ ) yielded 6-(4-methoxyphenyl) phenanthridine 1d (128 mg, 39\%) after column chromatography (alumina, $50 \%$ DCM in hexanes) as white crystals mp $144-145^{\circ} \mathrm{C}\left(\right.$ lit. $\left.^{7} 149-150{ }^{\circ} \mathrm{C}\right) \cdot v_{\max }(\mathrm{KBr}) / \mathrm{cm}^{-1} 1650$, $1575 ; \delta_{\mathrm{H}}\left(400 \mathrm{MHz}, \mathrm{CDCl}_{3}\right) 3.80\left(3 \mathrm{H}, \mathrm{s}, \mathrm{CH}_{3}\right), 6.63(2 \mathrm{H}, \mathrm{m}, \mathrm{Ar}-H), 6.81(1 \mathrm{H}, \mathrm{dd}, J 1.2,8.0, \mathrm{Ar}-$ $H), 6.88(1 \mathrm{H}, \mathrm{m}, \mathrm{Ar}-\mathrm{H}), 7.01(1 \mathrm{H}, \mathrm{m}, \operatorname{Ar}-H), 7.11(1 \mathrm{H}, \mathrm{m}, \mathrm{Ar}-H), 7.18(1 \mathrm{H}, \mathrm{dd}, J 1.2,8.0, \operatorname{Ar}-H)$, $7.38(2 \mathrm{H}, \mathrm{m}, \operatorname{Ar}-H), 7.55(2 \mathrm{H}, \mathrm{m}, \operatorname{Ar}-H), 7.71(1 \mathrm{H}, \mathrm{m}, \operatorname{Ar}-H) ; \delta_{\mathrm{C}}\left(100 \mathrm{MHz}, \mathrm{CDCl}_{3}\right) 56.7$ $\left(\mathrm{OCH}_{3}\right), 114.5$ (2 x Ar- $\left.\mathrm{CH}\right), 121.9$ (Ar-C), 124.2 (Ar- $\left.\mathrm{CH}\right), 126.9$ (Ar-C), $128.4(\mathrm{Ar}-\mathrm{CH}), 128.6$ (2 x Ar- $\mathrm{CH}), 128.9$ (2 x Ar- $\mathrm{CH}), 129.1$ (2 x Ar- $\mathrm{CH}), 129.3(\mathrm{Ar}-\mathrm{CH}), 130.2(\mathrm{Ar}-\mathrm{CH}), 132.3$ (Ar$C), 135.6$ (Ar- $C$ ), 137.4 (Ar- $C$ ), 138.5 (Ar-C), 161.7 (Ar-COMe), HRMS (FAB ${ }^{+}$) Found: $\left[\mathrm{M}+\mathrm{H}^{+}\right]$286.1233. $\mathrm{C}_{20} \mathrm{H}_{16} \mathrm{NO}$ requires 286.1232; $\mathrm{m} / \mathrm{z}\left(\mathrm{FAB}^{+}\right) 286\left(\left[\mathrm{M}+\mathrm{H}^{+}\right], 80 \%\right), 285(100)$, 254 (55), 241 (30).

6-(4-Chlorophenyl)phenanthridine (1e). ${ }^{7}$ Imidoyl-selanide $\mathbf{2 e}(80 \mathrm{mg}, 0.18 \mathrm{mmol})$ yielded 6(4-chlorophenyl)phenanthridine 1e (23 mg, 46\%) after column chromatography (alumina, 20\% DCM in hexanes) as white crystals mp $152-154{ }^{\circ} \mathrm{C}$ (lit. ${ }^{7} 160-161{ }^{\circ} \mathrm{C}$ ). $v_{\max }$ (neat) $/ \mathrm{cm}^{-1} 2358 ; \delta_{\mathrm{H}}$ $\left(400 \mathrm{MHz}, \mathrm{CDCl}_{3}\right) 7.54(2 \mathrm{H}, \mathrm{m}, \mathrm{Ar}-H), 7.64(1 \mathrm{H}, \mathrm{m}, \mathrm{Ar}-H), 7.70(3 \mathrm{H}, \mathrm{m}, \operatorname{Ar}-H), 7.77(1 \mathrm{H}, \mathrm{m}$, Ar- $H), 7.88(1 \mathrm{H}, \mathrm{m}, \mathrm{Ar}-H), 8.06(1 \mathrm{H}, \mathrm{dd}, J 0.8,8.4, \mathrm{Ar}-H), 8.23(1 \mathrm{H}, \mathrm{dd}, J 1.2,8.4, \mathrm{Ar}-H), 8.63$ $(1 \mathrm{H}, \mathrm{dd}, J 1.6,8.4, \mathrm{Ar}-H), 8.72(1 \mathrm{H}, \mathrm{d}, J 8.4, \mathrm{Ar}-H) ; \delta_{\mathrm{C}}\left(100 \mathrm{MHz}, \mathrm{CDCl}_{3}\right) 122.0(\mathrm{Ar}-\mathrm{CH}), 122.4$ $(\mathrm{Ar}-\mathrm{CH}), 123.8(\mathrm{Ar}-\mathrm{C}), 125.0(\mathrm{Ar}-\mathrm{C}), 127.2(\mathrm{Ar}-\mathrm{CH}), 127.3(\mathrm{Ar}-\mathrm{CH}), 128.5(\mathrm{Ar}-\mathrm{CH}), 128.7(2 \mathrm{x}$ $\mathrm{Ar}-\mathrm{CH}), 129.0(\mathrm{Ar}-\mathrm{CH}), 130.4(\mathrm{Ar}-\mathrm{CH}), 130.7$ (Ar- $\mathrm{CH}), 131.1$ (2 x Ar- $\mathrm{CH}), 133.5$ (2 x Ar-C), $134.9(\mathrm{Ar}-C), 143.8(\mathrm{Ar}-C-\mathrm{Cl}), 160.0(\mathrm{Ar}-C=\mathrm{N})$; HRMS $\left(\mathrm{FAB}^{+}\right)$Found: $\left[\mathrm{M}+\mathrm{H}^{+}\right] 290.0732$. 


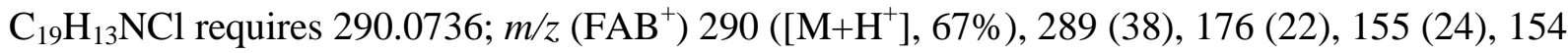
(100), 138 (25), 137 (48), 136 (61).

6-(4-Trifluoromethylphenyl)phenanthridine (1f). Imidoyl-selanide $2 \mathbf{f}$ (259 $\mathrm{mg}, 0.54 \mathrm{mmol}$ ) yielded 6-(4-trifluoromethylphenyl) phenanthridine 1f $(84 \mathrm{mg}, 48 \%)$ after column chromatography (alumina, 25\% DCM in hexanes) as white crystals mp 174-175 ${ }^{\circ} \mathrm{C} . v_{\max }$ $(\mathrm{KBr}) / \mathrm{cm}^{-1} 2368,1111 ; \delta_{\mathrm{H}}\left(400 \mathrm{MHz} \mathrm{CDCl}_{3}\right) 7.65(1 \mathrm{H}, \mathrm{dd}, J 1.2,7.2, \mathrm{Ar}-H), 7.73(1 \mathrm{H}, \mathrm{dd}, J 1.6$, 7.2, Ar- $H), 7.79(1 \mathrm{H}, \mathrm{dd}, J 1.6,7.2, \mathrm{Ar}-H), 7.87(5 \mathrm{H}, \mathrm{m}, \mathrm{Ar}-H), 8.03(1 \mathrm{H}, \mathrm{dd}, J 0.8,8.4, \operatorname{Ar}-H)$, $8.24(1 \mathrm{H}, \mathrm{m}, \mathrm{Ar}-H), 8.65(1 \mathrm{H}, \mathrm{dd}, J 1.6,8.0, \operatorname{Ar}-H), 8.74(1 \mathrm{H}, \mathrm{d}, J 8.4, \operatorname{Ar}-H) ; \delta_{\mathrm{C}}(100 \mathrm{MHz}$, $\left.\mathrm{CDCl}_{3}\right) 122.0(\mathrm{Ar}-\mathrm{CH}), 122.4(\mathrm{Ar}-\mathrm{CH}), 123.9(\mathrm{Ar}-\mathrm{C}), 124.9(\mathrm{Ar}-\mathrm{C}), 125.4\left(\mathrm{CF}_{3}\right), 125.5(2 \mathrm{x} \mathrm{Ar}-$ $C H), 125.6(\mathrm{Ar}-\mathrm{C}), 127.4(2 \mathrm{x} \mathrm{Ar}-\mathrm{CH}), 128.3(\mathrm{Ar}-\mathrm{CH}), 129.1(\mathrm{Ar}-\mathrm{CH}), 130.2(2 \mathrm{x} \mathrm{Ar}-\mathrm{CH}), 130.4$ $(\mathrm{Ar}-\mathrm{CH}), 130.9(\mathrm{Ar}-\mathrm{CH}), 133.5(\mathrm{Ar}-\mathrm{C}), 143.4(\mathrm{Ar}-\mathrm{C}), 143.7(\mathrm{Ar}-C), 159.7(\mathrm{Ar}-C=\mathrm{N})$; HRMS $\left(\mathrm{FAB}^{+}\right)$Found: $\left[\mathrm{M}+\mathrm{H}^{+}\right]$325.1004. $\mathrm{C}_{20} \mathrm{H}_{13} \mathrm{NF}_{3}$ requires $325.1000 ; \mathrm{m} / z\left(\mathrm{FAB}^{+}\right) 324\left(\left[\mathrm{M}+\mathrm{H}^{+}\right]\right.$, $100 \%), 323(21)$.

6-(4-Dimethylaminophenyl)phenanthridine (1g). Imidoyl-selanide $\mathbf{2 g}$ (109 mg, $0.24 \mathrm{mmol})$ yielded 6-(4-dimethylaminophenyl) phenanthridine $\mathbf{1 g}(16 \mathrm{mg}, 22 \%)$ after column chromatography (alumina, 25\% DCM in hexanes) as white crystals mp 155-157 ${ }^{\circ} \mathrm{C} . v_{\max }$ $(\mathrm{KBr}) / \mathrm{cm}^{-1} 1625 ; \delta_{\mathrm{H}}\left(400 \mathrm{MHz}, \mathrm{CDCl}_{3}\right) 3.00\left(6 \mathrm{H}, \mathrm{s}, \mathrm{N}\left(\mathrm{CH}_{3}\right)_{2}\right), 7.55(1 \mathrm{H}, \mathrm{dd}, J 1.2,7.2, \mathrm{Ar}-H)$, $7.70(1 \mathrm{H}, \mathrm{dd}, J 1.6,7.2$, Ar- $H), 7.80(1 \mathrm{H}, \mathrm{dd}, J 1.6,7.2, \operatorname{Ar}-H), 7.86(5 \mathrm{H}, \mathrm{m}, \mathrm{Ar}-H), 8.01(1 \mathrm{H}, \mathrm{dd}$, $J 0.8,8.4, \operatorname{Ar}-H), 8.30(1 \mathrm{H}, \mathrm{m}, \mathrm{Ar}-H), 8.66(1 \mathrm{H}, \mathrm{dd}, J 1.6,8.0, \mathrm{Ar}-H), 8.76(1 \mathrm{H}, \mathrm{d}, J 8.4, \operatorname{Ar}-H)$; $\delta_{\mathrm{C}}\left(100 \mathrm{MHz}, \mathrm{CDCl}_{3}\right) 40.7\left(2 \times \mathrm{CH}_{3}\right), 112.1$ (2 x Ar- $\left.\mathrm{CH}\right), 118.9(\mathrm{Ar}-\mathrm{CH}), 124.8(\mathrm{Ar}-\mathrm{C}), 124.9$ $(\mathrm{Ar}-\mathrm{CH}), 126.5$ (3 x Ar- $C \mathrm{H}), 128.1(\mathrm{Ar}-\mathrm{CH}), 129.9(\mathrm{Ar}-\mathrm{CH}), 130.1$ (2 x Ar- $\mathrm{CH}), 130.2$ (2 x Ar-

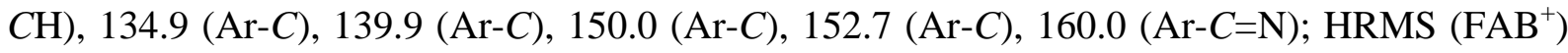
Found: $\left[\mathrm{M}+\mathrm{H}^{+}\right]$299.1548. $\mathrm{C}_{21} \mathrm{H}_{19} \mathrm{~N}_{2}$ requires 299.1548; $\mathrm{m} / z\left(\mathrm{FAB}^{+}\right) 299\left(\left[\mathrm{M}+\mathrm{H}^{+}\right], 100 \%\right), 120$ (56).

\section{General experimental procedure for the preparation of phenanthridine 1 by radical cyclisation}

To a solution of the imine 5 (1.0 equiv.) in $\mathrm{PhCl}(2 \mathrm{ml} / \mathrm{mmol})$ in a Young's tube was added ditert-butylperoxide (2.0 equiv.). The reaction vessel was deoxygenated by flushing with inert gas for $15 \mathrm{~min}$, then sealed and heated at $140{ }^{\circ} \mathrm{C}$ for $48 \mathrm{~h}$. The reaction mixture was allowed to cool to rt and quenched with $\mathrm{NaHSO}_{3}$ solution to remove any unreacted peroxide. The aqueous layer was then extracted with DCM $(3 \times 10 \mathrm{ml} / \mathrm{mmol})$. The combined organics were washed with water, brine, dried over $\mathrm{MgSO}_{4}$, filtered and concentrated under reduced pressure. The crude product was purified by column chromatography to yield phenanthridine product $\mathbf{1}$.

6-Phenylphenanthridine (1a). ${ }^{7}$ Imine 5a (350 mg, $\left.1.36 \mathrm{mmol}\right)$ yielded 6-phenyl phenanthridine 1a (138 $\mathrm{mg}, 40 \%)$ after purification. Characterisation data is the same as above.

6-(4-Tolyl)phenanthridine $\quad \mathbf{( 1 b )} .^{\mathbf{2 b}} \quad$ Imine $\quad \mathbf{5 b} \quad(320 \quad \mathrm{mg}, \quad 1.11 \quad \mathrm{mmol})$ yielded 6-(4tolyl)phenanthridine 1b (132 $\mathrm{mg}, 44 \%)$ after purification. Characterisation data is the same as above. 
6-(4-tert-butylphenyl)phenanthridine (1c). Imine 5c (350 mg, $1.12 \mathrm{mmol})$ yielded 6-(4-tertbutylphenyl)phenanthridine 1c (136 $\mathrm{mg}, 39 \%)$ after purification. Characterisation data is the same as above.

6-(4-Methoxyphenyl)phenanthridine (1d). ${ }^{7}$ Imine $\mathbf{5 d}$ (300 $\left.\mathrm{mg}, 1.04 \mathrm{mmol}\right)$ yielded 6-(4methoxyphenyl)phenanthridine $\mathbf{1 d}(148 \mathrm{mg}, 50 \%)$ after purification. Characterisation data is the same as above.

6-(4-Chlorophenyl)phenanthridine $\quad \mathbf{( 1 e )}^{{ }^{7}} \quad$ Imine $\mathbf{5 e}(300 \mathrm{mg}, 1.03$ mmol) yielded 6-(4chlorophenyl)phenanthridine 1e $(143 \mathrm{mg}, 48 \%)$ after purification. Characterisation data is the same as above.

6-(4-Trifluoromethylphenyl)phenanthridine (1f). Imine $\mathbf{5 f}$ (366 mg, $1.13 \mathrm{mmol}$ ) yielded 6-(4trifluoromethylphenyl)phenanthridine $\mathbf{1 f}(186 \mathrm{mg}, 51 \%)$ after purification. Characterisation data is the same as above.

6-(4-Dimethylaminophenyl)phenanthridine (1g). Imine $\mathbf{5 g}$ (350 mg, $1.17 \mathrm{mmol}$ ) yielded 6-(4dimethylaminophenyl)phenanthridine $\mathbf{1 g}$ (147 $\mathrm{mg}, 42 \%)$ after purification. Characterisation data is the same as above.

6-(4-Nitrophenyl)phenanthridine $\mathbf{( 1 h )})^{7} \quad$ Imine $\mathbf{5 h}(350 \mathrm{mg}, 1.16 \mathrm{mmol})$ yielded 6-(4nitrophenyl)phenanthridine $\mathbf{1 g}(160 \mathrm{mg}, 46 \%)$ after column chromatography (silica, 1:1 DCM:hexane) as white crystals mp 192-193 ${ }^{\circ} \mathrm{C}$ (lit. $\left.{ }^{7} 191-192{ }^{\circ} \mathrm{C}\right) . v_{\max }(\mathrm{KBr}) / \mathrm{cm}^{-1} 1550,1520$, 1350; $\delta_{\mathrm{H}}\left(400 \mathrm{MHz}, \mathrm{CDCl}_{3}\right) 7.69(1 \mathrm{H}, \mathrm{t}, J$ 8.0, Ar- $H), 7.79(3 \mathrm{H}, \mathrm{m}, \mathrm{Ar}-H), 7.98(3 \mathrm{H}, \mathrm{m}, \mathrm{Ar}-H)$, $8.29(1 \mathrm{H}, \mathrm{m}, \mathrm{Ar}-H), 8.47(2 \mathrm{H}, \mathrm{m}, \mathrm{Ar}-H), 8.67(1 \mathrm{H}, \mathrm{dd}, J 1.6,8.0, \mathrm{Ar}-H), 8.77(1 \mathrm{H}, \mathrm{d}, J 8.4$, Ar$H) ; \delta_{\mathrm{C}}\left(100 \mathrm{MHz}, \mathrm{CDCl}_{3}\right)$ 121.6 (Ar-CH), $122.7(\mathrm{Ar}-\mathrm{CH}), 123.6(\mathrm{Ar}-\mathrm{C}), 125.0(\mathrm{Ar}-\mathrm{C}), 125.5(2 \mathrm{x}$ $\mathrm{Ar}-\mathrm{CH}), 125.7(\mathrm{Ar}-\mathrm{C}), 127.6(2 \mathrm{x} \mathrm{Ar}-\mathrm{CH}), 128.1(\mathrm{Ar}-\mathrm{CH}), 128.7(\mathrm{Ar}-\mathrm{CH}), 130.1(2 \times \mathrm{Ar}-\mathrm{CH})$, $130.3(\mathrm{Ar}-\mathrm{CH}), 131.0$ (Ar- $C \mathrm{H}), 134.0(\mathrm{Ar}-C), 143.6$ (Ar- $C), 144.1(\mathrm{Ar}-C), 160.0$ (Ar- $C=\mathrm{N})$; HRMS $\left(\mathrm{FAB}^{+}\right)$Found: $\left[\mathrm{M}^{+}\right]$300.0896. $\mathrm{C}_{19} \mathrm{H}_{12} \mathrm{~N}_{2} \mathrm{O}_{2}$ requires 300.0899; $\mathrm{m} / z\left(\mathrm{FAB}^{+}\right) 300\left(\left[\mathrm{M}^{+}\right]\right.$, $90 \%), 122(100)$.

\section{References}

1. For example see:- (a) Li, D.; Zhao, B.; Sim, S.-P.; Li, T.-K.; Liu, A.; Liu, L. F.; LaVoie, E. J. Bioorg. Med. Chem. 2003, 11, 521. (b) Lynch, M. A.; Duval, O.; Sukhanova, A.; Devy. J.; MacKay, S. P.; Waigh, R. D.; Nabiev, I. Bioorg. Med. Chem. Lett. 2001, 11, 2643. (c) Ishikawa, T. Med. Res. Rev. 2001, 21, 61.

2. (a) Lysén, M.; Kristensen, J. L.; Vedsø, P.; Begtrup, M. Org. Lett. 2002, 4, 257. (b) Pawlas, J.; Begtrup, M. Org. Lett. 2002, 4, 2687. (c) Lysén, M.; Madden, M.; Kristensen, J. L.; Vedsø, P.; Zøllner, C.; Begtrup, M. Synthesis 2006, 3478.

3. (a) Mamalis, P.; Petrow, V. J. J. Chem. Soc. 1950, 703. (b) Buu-Hoï, N. P.; Jaquignon, P.; Long, C. T. J. Chem. Soc. 1957, 505.

4. Narasimham, N. S.; Chandrachood, P. S.; Shete N. R. Tetrahedron 1981, 37, 825. 
5. (a) Ishida, N.; Nakanishi Y.; Moriya T.; Murakami M. Chem. Lett. 2011, 40, 1047. (b) Peng, J.; Chen, T.; Chen, C.; Li, B. J. Org. Chem. 2011, 76, 9507. (c) Blanchot, M., Candito, D. A.; Larnaud, F.; Lautens M. Org. Lett. 2011, 13, 1486. (d) Candito, D. A.; Lautens, M. Angew. Chem. Int. Ed. 2009, 48, 6713. (e) Shabashov, D.; Daugulis, O. J. Org. Chem. 2007, 72, 7720. (f) Xie, C.; Zhang, Y.; Huang, Z.; Xu, O. J. Org. Chem. 2007, 72, 5431. (g) Shou, W.-G.; Yang, Y.-Y.; Wang, Y.-G. J. Org. Chem. 2006, 71, 9241.

6. (a) Katritzky, A. R.; Yang B. Z. J. Heterocycl. Chem. 1996, 33, 607. (b) Rosa, A. M.; Lobo A. M.; Branco P. S.; Prabhakar, S.; Pereira A. M. D. L. Tetrahedron 1997, 53, 269. (c) Nakanishi, T.; Suzuki, M.; Mashiba, A.; Ishikawa, K.; Yokotsuka T. J. Org. Chem. 1998, 63, 4235. (d) Ellis, M. J.; Stevens, M. F. G. J. Chem. Soc., Perkin Trans. 1 2001, 3180. (e) Portela-Cubillo, F.; Scott, J. S.; Walton, J. C. J. Org. Chem. 2008, 73, 5558.

7. Leardini R.; Tundo A.; Zanardi G.; Pedulli G. F. Synthesis 1985, 107-110.

8. For recent examples see:- (a) Mondal, P.; Thander L; Chattopadhyay, S. K. Tetrahedron Lett. 2012, 53, 1328. (b) Jana, G. P.; Mukherjee, S.; Ghorai, B. K. Synthesis 2010, 3179. (c) Zhang, L.; Ang, G. Y.; Chiba, S. Org. Lett. 2010, 12, 3682. (d) Xi, J.; Dong, Q.-L.; Liu G.S.; Wang, S.; Chen, L., Yao, Z.-J. Synlett 2010, 1674. (e) Budén, M. E.; Dorn, V. B.; Gamba, M.; Pierini, A. B.; Rossi, R. A. J. Org. Chem. 2010, 75, 2206. (f) Youn, S. W.; Bihn J. H. Tetrahedron Lett. 2009, 50, 4598. (g) Jana, G. P.; Ghorai B. K. Lett. Org. Chem. 2009, 6, 372 .

9. Bowman, W. R.; Lyon, J. E.; Pritchard, G. J. Synlett 2008, 2169.

10. Christie, S. D. R.; Davoile, R. J.; Elsegood, M. R. J.; Fryatt, R.; Jones, R. C. F.; Pritchard, G. J. Chem. Commun. 2004, 2474; Ellart, E. A.; Christie, S. D. R.; Pritchard, G. J.; Elsegood, M. R. J. Chem. Commun. 2009, 7339.

11. For example see:- Irlapati, N. R.; Baldwin, J. E.; Adlington, R. M.; Pritchard, G. J.; Cowley, A. R. Tetrahedron 2006, 62, 4603; Adlington, R. M.; Baldwin, J. E.; Mayweg A. V. W.; Pritchard, G. J. Org. Lett. 2002, 4, 3009; Adlington, R. M.; Baldwin, J. E.; Williams A. J.; Watkin, D. J. Org. Lett. 1999, 1, 1937; Irlapati, N. R.; Baldwin, J. E.; Adlington R. M.; G. J. Pritchard, Org. Lett. 2003, 5, 2351.

12. For examples see:- Adamo, M. F. A.; Adlington, R. M.; Baldwin, J. E.; Pritchard G. J.; Rathmell, R. E. Tetrahedron 2003, 59, 2197; Baldwin, J. E.; Fryer A. M.; Pritchard, G. J. Bioorg. Med. Chem. Lett. 2000, 10, 309; Adlington, R. M.; Baldwin, J. E.; Catterick, D.; Pritchard, G. J. Chem. Commun. 1997, 1757.

13. Pedersen, J. M.; Bowman, W. R.; Elsegood, M. R. J.; Fletcher, A. J.; Lovell, P. J. J. Org. Chem. 2005, 70, 10615; Bowman, W. R.; Elsegood, M. R. J.; Stein, T; Weaver, G. W. Org. Biomol. Chem. 2007, 5, 103; Allin, S. M.; Barton W. R. S.; Bowman, W. R.; Bridge, E.; Elsegood, M. R. J.; McInally, T.; McKee, V. Tetrahedron 2008, 64, 7745.

14. Bowman, W. R.; Fletcher, A. J.; Pedersen, J. M.; Lovell, P. J.; Elsegood, M. R. J.; Lopez, E. H.; McKee, V.; Potts, G. B. S. Tetrahedron 2007, 63, 191.

15. Fujiwara S.; Toyofuku M.; Kuniyasu H.; Kambe N. Pure Appl. Chem. 2010, 82, 565.

16. Newcomb, M; Esker, J. L. Tetrahedron Lett. 1991, 32, 1035. 
17. (a) Li, J. J.; Gribble, G. W. Palladium in Heterocyclic Chemistry; Pergamon: Oxford, 2000. (b) Tsuji, J. Palladium Reagents and Catalysts; Wiley: Chichester, 2004.

18. Bowman, W. R.; Bridge, C. F.; Brookes, P.; Cloonan, M. O.; Leach, D. C. J. Chem. Soc., Perkins Trans. 1 2002, 58.

19. Bowman, W. R.; Storey, J. M. D. Chem. Soc. Rev. 2007, 36, 1803. 